\title{
Investigation of the effect of grain clusters on fatigue crack initiation in polycrystals
}

\author{
Y. Guilhem ${ }^{\mathrm{a}, \mathrm{c}, *}$, S. Basseville ${ }^{\mathrm{b}}$, F. Curtit ${ }^{\mathrm{c}}$, J-M. Stéphan $^{\mathrm{c}}$, G. Cailletaud $^{\mathrm{a}}$ \\ ${ }^{a}$ MINES ParisTech, Centre des Matériaux, CNRS UMR 7633, BP 87, 91003 Evry Cedex, France \\ ${ }^{b}$ Université de Versailles Saint-Quentin, Laboratoire des Systèmes d'Ingénierie de Versailles, 45 avenue des Etats-Unis, 78035 Versailles, \\ France \\ ${ }^{c}$ EDF RED, Département Matériaux et Mécanique des Composants, Avenue des Renardières, Ecuelles, 77818 Moret-sur-Loing Cedex, \\ France
}

\begin{abstract}
Fatigue crack initiation in ductile alloys like austenitic stainless steels is mainly due to the occurrence of localized deformation in persistent slip bands (PSB). The presence of PSB is classically related to the orientation of the surface grains. In fact, the local fields in a grain does not depend on the local orientation only. The aim of the present paper is to investigate the consequences of this observation, and to propose an analysis, where the neighborhood of the grain also plays a significant role. The study is made on a 316 stainless steel. Finite element computations using a crystal plasticity model are performed to simulate an aggregate submitted to a cyclic tension-compression loading. Various configurations of grain orientations ("clusters") are studied at the free surface of the aggregate. A statistical analysis of the results is carried out to extract significant information concerning the local strain and stress fields, including the most critical arrangements of grain orientations. The introduction of local fields in classical fatigue life prediction models provides an explanation of the experimental scatter.
\end{abstract}

Key words: polycrystal, crystal plasticity, finite element analysis, fatigue prediction, short cracks

\section{Introduction}

Fatigue crack initiation is classically predicted by macroscopic models, where the critical variables are functions of macroscopic stress and strain. The various regimes associated with crack propagation were characterized a long time ago (see for instance 11). Stage I (initiation) and stage II (propagation) were first introduced. Then crack nonpropagatation was characterized through the fatigue limit [2]. However, it has been shown that initiation and micro-propagation can occur below this criterion as long as the crack does not cross microstructural barriers [3]. The propagation rate is then affected by the local geometry, and cannot be predicted by linear elastic fracture mechanics using global variables [4, 5. The very early stages of initiation and propagation of Microstructurally Short Cracks (MSC), with lengths on the order of a few grains, are mainly governed by the material microstructure. Indeed, in the case of FCC metals, surface roughness, grain or phase boundaries, inclusions, plastic incompatibilities and persistent slip bands (PSB) are the main causes of MSC nucleation. The elastic-plastic anisotropy is a source of deviation of local crack growth driving force. In High Cycle Fatigue (HCF), initiation occurs generally on the component surface [6] and this phase can represent more

*Corresponding author. Address: MINES ParisTech, Centre des Matériaux, CNRS UMR 7633, BP 8791003 Evry Cedex, France; Tel.: +33-1-60-76-30-67; fax: +33-1-60-76-31-50.

Email addresses: yoann.guilhem@ensmp.fr (Y. Guilhem) than $80 \%$ of the total component life. Considering a clean and finely polished metal specimen, the local mechanisms responsible for MSC initiation and micro-propagation are restricted to PSBs or elastic anisotropy and plastic incompatibilities at boundaries. Thus, they are defined on a microscale and they are closely related to grain orientation.

Intrusion and extrusions related to slip planes have been observed on the specimen surface after fatigue loading. A crack can initiate at the base of these steps once their height reaches about $1 \mu \mathrm{m}$. A model introduced by Essmann et al. that is based on dislocation movement and annihilation explains this local mechanism [7. Removing the induced surface roughness by periodical polishing increases fatigue life as shown on single crystals by Basinsiki et al. 8]. At each cycle, oxidation takes place on the surface extrusion and the oxides are absorbed during the reverse loading phase. This will induce slip irreversibility, decohesion hence crack can initiate easily from this singularities. This environment effect has been highlighted by experimental fatigue testing in air and vacuum where fatigue life is longer [9]. Since MSC are related to PSB and surface extrusion, their direction is governed by crystal plasticity and follow slip planes containing slip systems in the surface grains. Crack paths have been correlated to crystal orientation measured by Electron BackScattered Diffraction (EBSD). Based on Schmid factor values and neglecting neighborhood influence, studies first showed that surface extrusions are related to primary slip systems acti- 
vation 10. The dependence of initiation and propagation sites location on plastic strain amplitude has been highlighted [11, 12].

The microstructure is also the source of a competition between transgranular and intergranular cracks [13]. On the one hand, in the case of low amplitude fatigue of polycrystalline materials, transgranular cracks are predominant. Certain misorientations can decrease the transgranular crack growth and lead to intergranular crack growth [14]. On the other hand, at higher strains, a strong misorientation can cause initiation on the surface along grain boundaries [15, 16]. The parameters like crystal orientation, grain size and shape have to be taken into account to reproduce local strain heterogeneities which govern crack initiation. Taking account of all these observations (see also for instance [17, 18]), it is now useful to complement the experimental data by relevant numerical investigations. To have a better understanding of the local mechanisms influencing crack formation, the study is focused on the orientation of the grain with mesoscopic modeling. The purpose of this paper is to get an improved understanding of the local stress and strain fields. Simulation of a real 3D microstructure with hundreds of finely meshed grains including a real crack loaded for several thousands of cycles is still out of reach; this is why authors develop representative examples on reduced microstructures. Two dimensional meshes are used for instance on single crystal, bicrystals [19] and polycrystals [20, 21]. As an example, 27 grains are used in [22] and 12 grains are used in [23. Such a low number of grains does not correspond to a representative volume or surface element, so that the conclusions are qualitative only. It remains that the damaging effect arising from boundaries between hard and soft region is actively studied in the literature [22, 24]. 2D Meshes using Voronoï tessellation, are introduced to investigate the propagation of a microcrack [25, 26]. Using a crystal plasticity model, they showed that the crack propagation was directed by two primary slip planes. The crack tip opening exhibits large changes due to the orientation of the grains in the vicinity [27, 28. It is also shown that cracks will preferably initiate from the "soft" grains where cracks first start 29. So, the investigations are more directed toward the orientation of each grain, or to grain boundary misorientation. The aim of this paper is to introduce a new element in the discussion, pointing out the fact that stress redistribution operates on grain clusters more than on individual grains or grain pairs. Stress distributions are rather complex, and their development must be studied in a statistical framework. This was for instance considered in a recent paper 30 where authors perform dozens of FE computations, to characterize the tails of the curves. Nevertheless, this study uses a ruled mesh, the grains represented by a Voronoï tessellation being only mapped by a multimaterial element procedure. It does not conform grain boundaries. In the present paper, FEA is used to study the effect of particular grain "clusters" located at the free surface of the aggregate to show how the different grains interact with each other. A statistical study is performed to characterize the influence of the neighborhood on soft or hard grains, oriented for easy or hard slip. The combination of clustering effect and a statistical treatment has not been tried previously in the literature according to authors' best knowledge. Such a study is significant only with a large number of FE samples. This is why $2 \mathrm{D}$ computations have been performed. It has been shown that they could provide realistic results if compared with real experiments 22]. The multiaxial state of stress will be studied. A crystal plasticity model with nonlinear kinematic hardening is introduced in a regular $2 \mathrm{D}$ mesh which conforms grain boundaries. The results from microscale are finally used in a classical fatigue life prediction model to predict local crack initiation.

The paper is organized as follows. First, the numerical model is presented (constitutive equations, FE mesh and boundary conditions). Next, a careful study of the stress redistribution is made for the case of randomly oriented grains and then for specific local arrangements. Finally, the local fields are introduced in a very simple crack initiation rule to predict crack initiation.

\section{Description of the numerical model}

\subsection{Crystal plasticity model}

A crystal plasticity model is introduced in the finite element code ZeBuLoN [31. Small strain assumption is used. This assumption is reasonable, since, in our past experience, the amount of rotation of a slip plane is around $1^{\circ}$ for $1 \%$ macroscopic strain. In our computations, there is no local ratchetting, since the loading is symmetric and the local strain remains lower than 5\%. Each grain is considered as a single crystal and the displacement fields are supposed to be continuous at grain boundaries. Stress discontinuities can therefore appear at these sites. The partition of the strain rate tensor introduces an elastic and a viscoplastic part:

$$
\underset{\sim}{\dot{\varepsilon}}={\underset{\sim}{\dot{\varepsilon}}}^{e}+{\underset{\sim}{\dot{\varepsilon}^{p}}}^{p}{\underset{\sim}{\mathbf{C}}}^{-1}: \underset{\sim}{\dot{\sigma}}+{\underset{\sim}{\dot{\varepsilon}^{p}}}^{p}
$$

Cubic elasticity is defined by the fourth order stiffness tensor $\underset{\approx}{\mathbf{C}}$, so that elasticity itself is the source of residual intergranular stresses. As confirmed by the literature, this effect is important for small strains, as long as plastic strains do not become predominant. Its introduction is mandatory to reach a good agreement with experimental results 32. We decided to introduce cubic elasticity to capture the stress heterogeneity at the onset of plastic flow. The resolved shear stress $\tau^{s}$ is computed on slip system $s$ by means of the orientation tensor $\mathbf{m}^{s}$ :

$$
\begin{gathered}
\tau^{s}=\underset{\sim}{\sigma}: \underline{\sim}^{s} \\
\text { with } \underline{\sim}^{s}=\frac{1}{2}\left(\underline{\mathbf{l}}^{s} \otimes \underline{\mathbf{n}}^{s}+\underline{\mathbf{n}}^{s} \otimes \underline{\mathbf{l}}^{s}\right)
\end{gathered}
$$

Here, $\underline{\mathbf{n}}^{s}$ is the normal to the slip plane, and $\underline{\mathbf{l}}^{s}$ is the slip direction. The viscoplastic slip rate $\dot{\gamma}^{s}$ is given by a power 
function of the resolved shear stress, then the viscoplastic strain rate tensor is defined as the sum of the contributions of all the slip systems.

$$
\begin{gathered}
\dot{v}^{s}=\left\langle\frac{\left|\tau^{s}-x^{s}\right|-R_{0}-r^{s}}{K}\right\rangle^{n} \\
\dot{\gamma}^{s}=\dot{v}^{s} \operatorname{sign}\left(\tau^{s}-x^{s}\right) \\
\dot{\sim}^{p}=\sum_{s} \dot{\gamma}^{s} \mathbf{m}^{s}
\end{gathered}
$$

where $K$ and $n$ are the parameters which define viscosity, $R_{0}$ is the critical resolved shear stress, $x^{s}$ and $r^{s}$ are respectively the kinematic hardening and the isotropic hardening variables. These variables depend on two state variables, namely $\alpha^{s}$ and $\rho^{s}$ as described by equations 7 to 10 . This framework offers a unique set of active slip systems, and avoids complex procedures attached to the definition of slip activity for the time independent plastic case [33].

$$
\begin{gathered}
x^{s}=c \alpha^{s} \\
r^{s}=b Q \sum_{r} h_{s r} \rho^{r} \\
\dot{\alpha}^{s}=\left(\operatorname{sign}\left(\tau^{s}-x^{s}\right)-d \alpha^{s}\right) \dot{v}^{s} \\
\dot{\rho}^{s}=\left(1-b \rho^{s}\right) \dot{v}^{s}
\end{gathered}
$$

The material parameters are $c, d$ (kinematic hardening), $Q, b$ (isotropic hardening). The interaction matrix $h_{s r}$ introduces self-hardening (diagonal terms) and latent hardening between the different slip systems. All the values used in the FE simulation are listed in Tab. 1. With these prescribed values, the viscous effect is negligible.

The purpose of the paper is to exhibit the effect of clusters of grains on the initiation of MSC. A series of extreme situations have then been created, in order to provide the best illustration of the phenomena. Since the paper is restricted to a numerical study, it was decided to perform $2 \mathrm{D}$ computations only. A generalized plane strain assumption is used, and only one active slip system is allowed in each grain. Even though this is a strong idealization of the real material behavior, Zouhal et al. showed that in polycrystalline materials the number of active slip systems tends to 1 after a certain number of cycles 34. Furthermore, it has also been shown that in the case of HCF single slip activation occurs at the surface during the crack initiation phase [35, which is the main purpose of this paper. As displayed in Fig. 1 , the vectors $\underline{\mathbf{n}}$ and $\underline{\mathbf{l}}$ are both located in the plane $\left(x_{1}, x_{2}\right)$, and $\theta$ is the angle between $x_{1}$ and the slip direction, so that:

$$
\underline{\mathbf{l}}=(\cos \theta \sin \theta 0) \quad \underline{\mathbf{n}}=(-\sin \theta \cos \theta 0)
$$

According to this notation, a grain is called "hard" for $\theta=0^{\circ}$ (zero resolved shear stress if computed with the macroscopic stress tensor) and "soft" for $\theta=45^{\circ}$ (maximum resolved shear stress if computed with the macroscopic stress tensor).

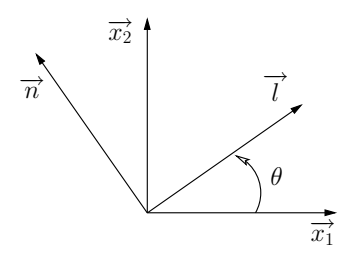

Figure 1: Single slip system and definition of crystallographic orientation $\theta$.

The accumulated viscoplastic strain $p$ and its related grain average $\langle p\rangle^{g}$, which are regarded as primary variables in this paper, are written as:

$$
\begin{gathered}
p=\sum_{s} \int_{t_{0}}^{t} \dot{v}^{s} d t \\
\langle p\rangle^{g}=\frac{1}{V} \int_{V} p d V
\end{gathered}
$$

\subsection{Fatigue criteria}

Three different criteria are introduced taking account of the local state and depending on the slip systems activity. The reason why we are choosing these three models is that we wish to have a purely strain dependent criterion, a purely stress dependent criterion, and a mixed solution.

(i) In the criterion suggested by Fatemi and Socie 36, the plane with the maximum shear strain amplitude is critical. The fatigue life prediction is done by means of the maximum shear strain amplitude and the maximum normal stress

$$
\Delta \gamma_{S F}=\max _{s}\left(\frac{\Delta \gamma^{s}}{2}\left(1+k_{S F} \max _{t} \frac{\sigma_{n}^{s}(t)}{\sigma_{y}}\right)\right)
$$

where $\Delta \gamma^{s}$ is the maximum range of shear strain on the slip system $s, \sigma_{n}^{s}$ is the normal stress to the slip plane of the slip system $s, \sigma_{y}$ is the initial value of the critical resolved shear stress (i.e. $R_{0}$ ) and $k_{S F}$ is a material parameter.

(ii) The stress dependent criterion is inspired by the classical HCF model proposed by Dang Van [37]. The original formulation introduces an equivalent stress

$$
\sigma_{D V}=\max _{s}\left(\max _{t}\left(\tau_{r}^{s}(t)+k_{D V} P(t)\right)\right)
$$

where $P$ is the hydrostatic pressure, $\tau_{r}^{s}$ the resolved shear stress recentered on the slip system $s$

$$
\tau_{r}^{s}(t)=\tau^{s}(t)-\frac{1}{2}\left(\max _{t}\left(\tau^{s}(t)\right)+\min _{t}\left(\tau^{s}(t)\right)\right)
$$

$k_{D V}$ is a material parameter.

(iii) the accumulated viscoplastic strain $p$ 


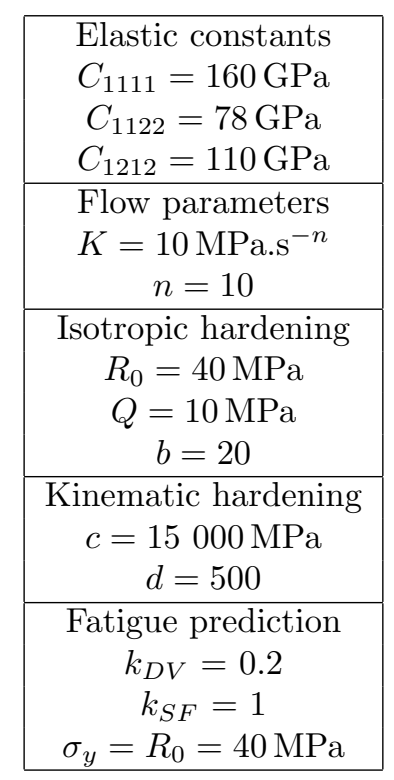

Table 1: Material parameters.

\subsection{Finite element mesh and boundary conditions}

The mesh consists of a polycrystalline aggregate made of 71 grains, 10,561 nodes and 5178 generalized plane strain elements with a quadratic interpolation. All the grains have a regular hexagonal shape, so that there is no trouble produced by a variable shape or size. As shown in Fig. 2 , the grains at the boundaries can be "open" (a hexagon cut by the surface) or "closed" (a full hexagon touches the surface). The differences between these two types of grains will be investigated later.

Symmetry conditions are applied at the bottom $\left(u_{2}=\right.$ $0)$ and the right side $\left(u_{1}=0\right)$ of the mesh, as displayed in Fig. 2, A cyclic displacement corresponding to a $\pm 2 \%$ average strain is applied to the top of the aggregate for 10 cycles. The left edge is a free surface.

\section{Results and discussion}

\subsection{Random aggregates}

Before considering specific clusters, a preliminary study is made on realizations with randomly oriented grains. Its purpose is to illustrate the polycrystal effect in the aggregate. A large number of FE analyses have been made (800 different cases) in order to elaborate a statistical view towards the aggregate response. The accumulated viscoplastic strain in each grain, $p$, is computed, then its volume average is calculated for each class of crystallographic orientation. The result is reported in Fig. 3, where the value obtained for the polycrystal is compared to the value obtained for the single crystal, which is obtained by applying the same loading on a single grain mesh. In this last case, for a tensile component $\sigma$, the resolved shear stress on the unique slip system is $\sigma \sin (2 \theta) / 2$, so that there is no plastic strain when $\theta$ is close to $0^{\circ}$ or $90^{\circ}$, since the Schmid factor tends to zero. This is no longer true for
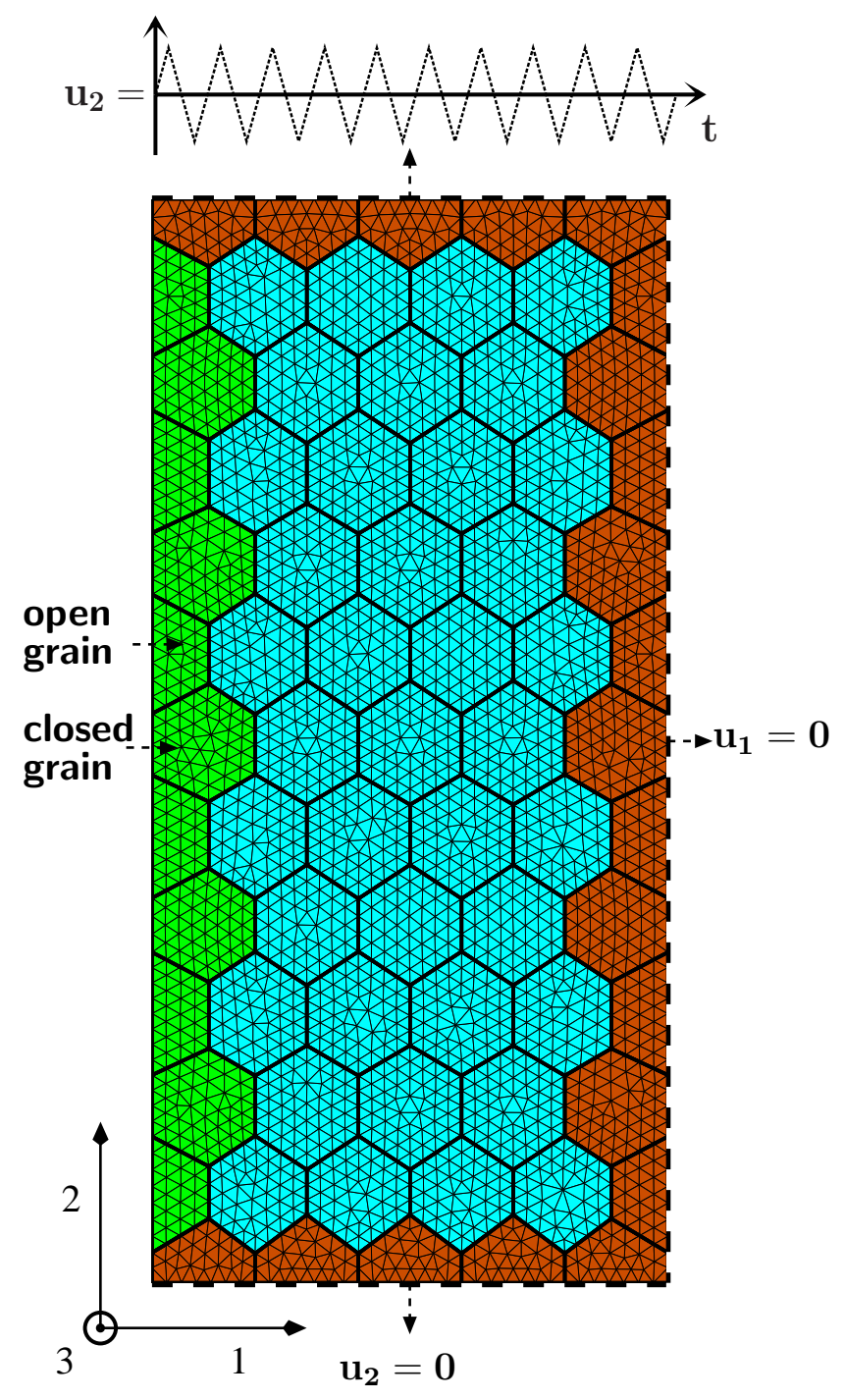

Core grains

Grains involved in BC's

Surface grains

Figure 2: Mesh and boundary conditions. 


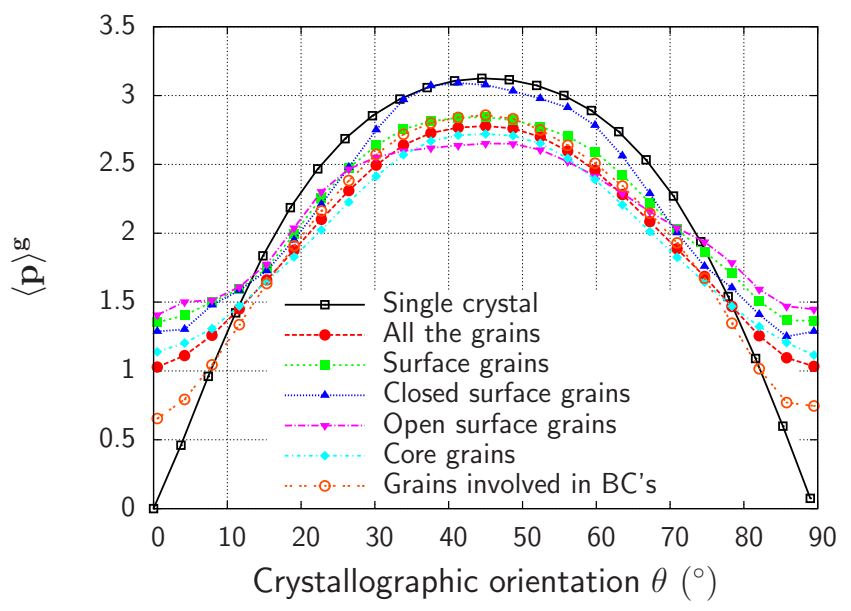

Figure 3: Accumulated plastic strain versus grain orientation for different types of grains.

the polycrystalline aggregates. Different types of grains (various locations, various geometries and relative sizes...) are examined and compared to the single crystal simulations. The single crystal data are normalized to get the same average value over all orientation as in the case of the polycrystal. All the curves are plotted on the same graph to summarize the results in Fig. 3. They are plotted separately in Fig. 4 to show the standard deviation for each class of crystallographic orientation.

When compared to the single crystal case, the distribution of the accumulated plastic shear strain of the aggregates (taken in average, for each class of grain orientation) presents a smaller range, as shown in Fig. 4a. On one hand, due to the multiaxiality of the local stress field that is a consequence of the stress redistribution, it becomes possible to get non zero $p$ even when $\theta=0^{\circ}$. The value obtained for $0^{\circ}$ and $90^{\circ}$ is quite high (more than one third of the maximum value, obtained for $\left.\theta=45^{\circ}\right)$. On the other hand, for soft grains $\left(\theta=45^{\circ}\right), p$ is lower than the single crystal (Fig. 4a). The curves are symmetric with respect to $\theta=45^{\circ}$, since one could exchange the role of $\underline{\mathbf{n}}^{s}$ and $\underline{\mathbf{l}}^{s}$, due to the small strain assumption.

By restricting the plot either to surface grains (Fig. 4b) or to core grains (Fig. 4e), the curves have still the same shape. Nevertheless, it can be observed that the scatter is larger for the surface grains. The number of surface grains in the mesh is 11 . Since 800 FEA have been made, this allows us to compute the average of plastic strain on 8800 surface grains, so that the resulting number is statistically valid. It can then be concluded that the scatter is intrinsically larger at the surface of the aggregates. This conclusion was already pointed out in previous studies on $3 \mathrm{D}$ aggregates 38 . This is due to the free boundary condition which enhances heterogeneities. The opposite is true for grains affected by boundary conditions (Fig. 4f). Indeed, they present a weaker cumulated viscoplastic strain when $\theta$ is close to $0^{\circ}$ and $90^{\circ}$. The decrease of $p$ for $\theta$ (a) Hard grains $\left(\theta<1^{\circ}\right.$ and $\left.\theta>89^{\circ}\right)$.

\begin{tabular}{ccccc}
\hline & \multicolumn{3}{c}{$p$} \\
\cline { 2 - 5 } Type & Min & Mean & Max & Std deviation \\
\hline All & 0.0013 & 1.0615 & 5.3454 & 1.3310 \\
Surface & 0.0013 & 1.3066 & 5.3454 & 1.6670 \\
Closed & 0.0138 & 1.2273 & 3.9266 & 1.5448 \\
Open & 0.0013 & 1.4217 & 5.3454 & 1.8139 \\
Core & 0.0186 & 1.1366 & 4.1086 & 1.3427 \\
BC & 0.0138 & 0.7317 & 3.9995 & 1.0229 \\
\hline
\end{tabular}

(b) Soft grains $\left(44^{\circ}<\theta<46^{\circ}\right)$.

\begin{tabular}{ccccc}
\hline & \multicolumn{3}{c}{$p$} \\
\cline { 2 - 5 } Type & Min & Mean & Max & Std deviation \\
\hline All & 0.2052 & 2.9489 & 9.2971 & 3.3312 \\
Surface & 0.2052 & 2.9622 & 8.2779 & 3.4329 \\
Closed & 0.2052 & 3.2016 & 8.2779 & 3.6571 \\
Open & 0.3923 & 2.6689 & 7.9762 & 3.1020 \\
Core & 0.5503 & 2.8254 & 6.7021 & 3.1023 \\
BC & 0.2052 & 3.0787 & 9.2971 & 3.5734 \\
\hline
\end{tabular}

Table 2: Statistical data of accumulated plastic strain $p$ at Gauss point scale.

$=0^{\circ}$ with respect to Fig. $4 \mathrm{a}$ is about $50 \%$. So, these initial observations are enough to confirm the effects of the surrounding grains on the local redistribution.

A more careful observation is then made, concerning the shape of the surface grains. Closed grains are considered in Fig. 4c and open grains in Fig. 4d. The first ones are represented by a full hexagon, meanwhile the second ones are only made by one half. First, the scatter observed in Fig. $4 \mathrm{~b}$ for all the surface grains is present for both classes of grains. Nevertheless, there is a clear effect of the shape of the grains. Indeed, closed grains tend to follow the same behavior as the other grains around $0^{\circ}$ but present a higher value of $p$ near $45^{\circ}$. For some cases, values larger than those obtained for the single crystal are found. These grains are the most deformed in the distribution. On the other hand, the distribution plotted for open grains is quite flat. They have the highest values of $p$ for $\theta=0^{\circ}$ or $90^{\circ}$, and very low values for $\theta=45^{\circ}$. In fact, they are unable to "impose their own behavior", and follow the influence of the neighborhood.

A statistical approach on the Gauss point values inside the soft and hard grains is also given. The analysis of cumulative distributions (see Fig. 5) and statistical data (given in Tab.2) confirms that surface grains are very often the location of largest slip values. It reveals also, on the one hand, that through all hard grains, the open surface ones are the most critical. On the other hand, through soft grains, the most critical are the closed surface ones.

This preliminary study confirms that the highest variability around the mean value is obtained at the surface. As a consequence, the maximum values are also reached at the surface for all the variables (in terms of stress or strain). A straightforward consequence is that it will be 


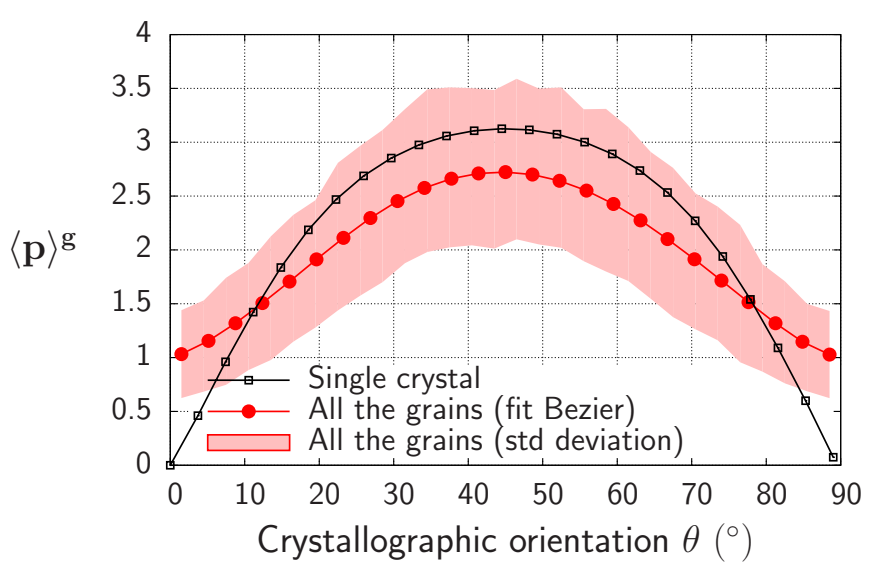

(a) All the grains

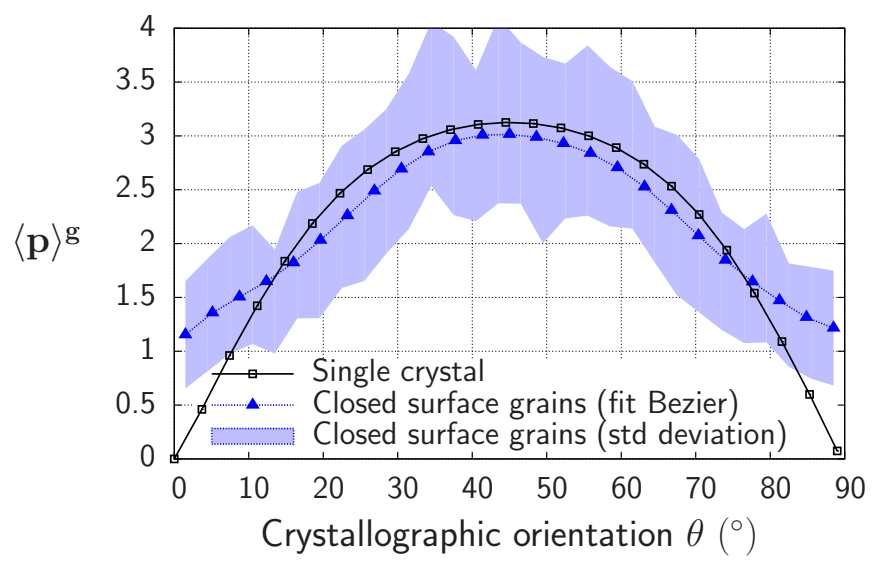

(c) Closed surface grains

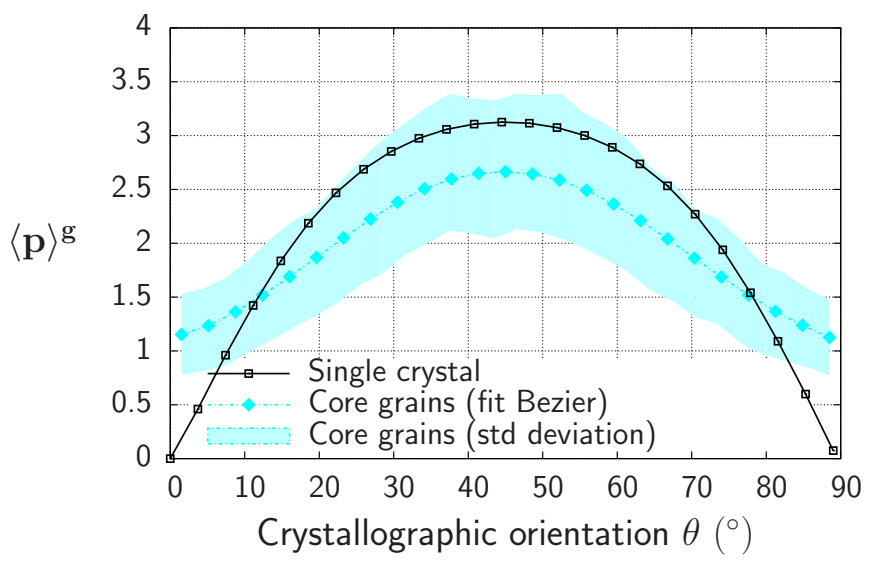

(e) Core grains

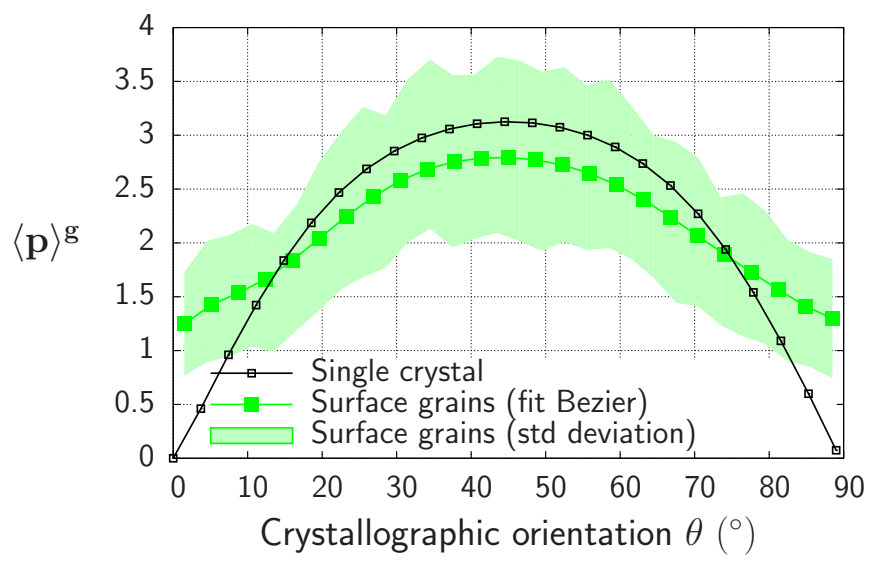

(b) Surface grains

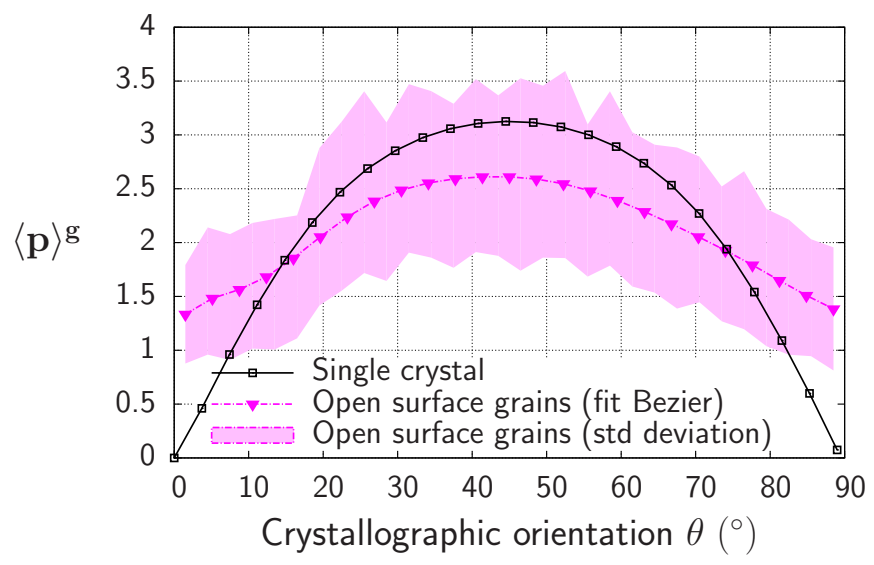

(d) Open surface grains

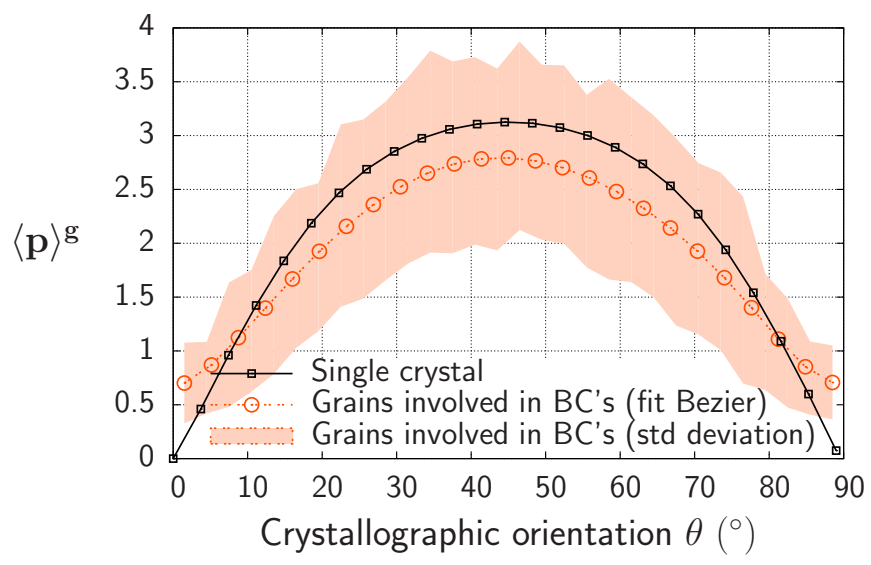

(f) Grains involved in BC's

Figure 4: Plastic behavior of different types of grains and comparison with single crystal. 


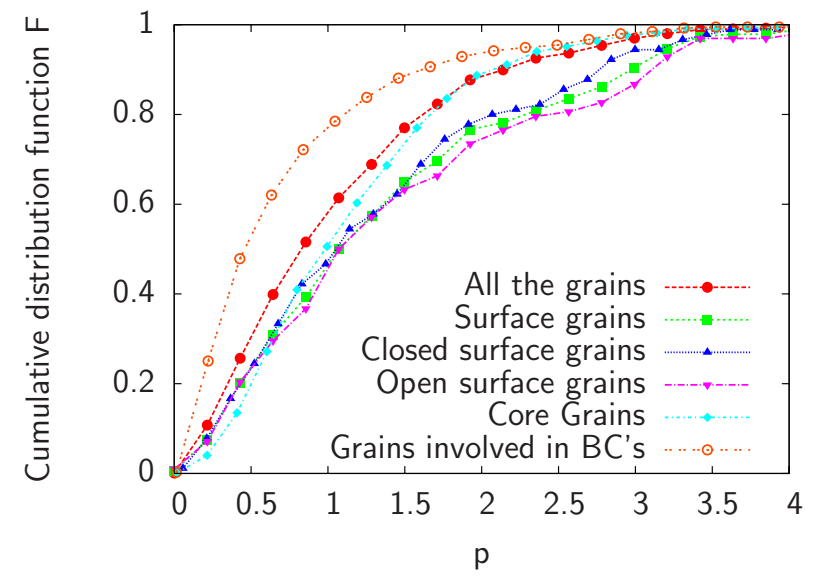

(a) Hard grains $\left(\theta<1^{\circ}\right.$ and $\left.\theta>89^{\circ}\right)$.

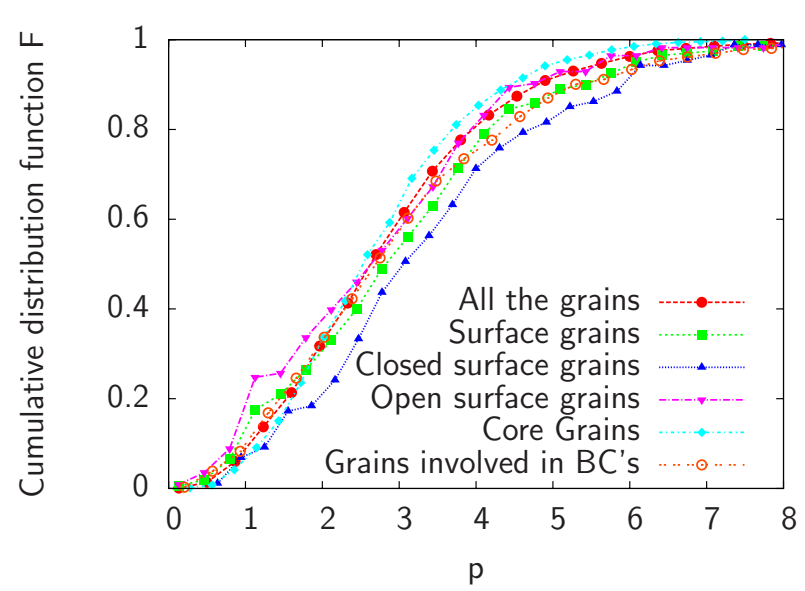

(b) Soft grains $\left(44^{\circ}<\theta<46^{\circ}\right)$.

Figure 5: Cumulative distribution functions of accumulated plastic strain for different types of grains.

the place for crack initiation. In the next section, the study focuses on groups of grains at the free surface, to explore the most critical cluster configurations.

\subsection{Clustering effect}

Different sets of orientations corresponding to extreme configurations are tested to put forward the influence of the crystallographic orientation. They correspond to extreme cluster configuration with soft or hard grains inside a hard or soft matrix. Many clusters were studied, but only two geometric arrangements are retained. For each of them, the grain in the middle is characterized by an angle $\theta_{1}$, meanwhile all the surrounding grains have an angle $\theta_{2}$ (Fig. 6). The arrangement shown in Fig. 6a has a hexagonal ("closed") grain surrounded by seven grains. Figure 6b shows the case of an "open" grain surrounded by three grains. The values taken by angle $\theta_{1}$ is either $0^{\circ}$ or $45^{\circ}$. The angle $\theta_{2}$ is then respectively equal to $45^{\circ}$ or $0^{\circ}$. In addition, a series of computations are performed with random values for $\theta_{2}$. Eight types of clusters are then defined. Each name is built by gathering the information for the middle grain, and a letter for the shape ( $\mathrm{C}$ for closed; O for open), a number for the orientation (0 or 45) and another number or letter for the orientation of the external grains $(0,45$, or $\mathrm{R}$ for random). The $*$ symbol is used as a wild card to replace each of these symbols (for instance $*-45-0$ refers to both $\mathrm{C}-45-0$ and $\mathrm{O}-45-0$ clusters, and $\mathrm{C}-45-*$ corresponds to $\mathrm{C}-45-0$ and $\mathrm{C}-45-45$ and $\mathrm{C}-$ $45-\mathrm{R})$. All the other grains in the aggregate are randomly oriented, and a series of different realizations have been made, to reflect the statistical aspect of the problem. So, for instance a hard $\left(0^{\circ}\right)$ open central grain within a soft surrounding will be noted $\mathrm{O}-0-45$.

\subsubsection{Study of the strain fields}

The prescribed macroscopic strain is $\pm 2 \%$. The accumulated slip in each cycle is zero if $\theta=0^{\circ}$ and about 0.15
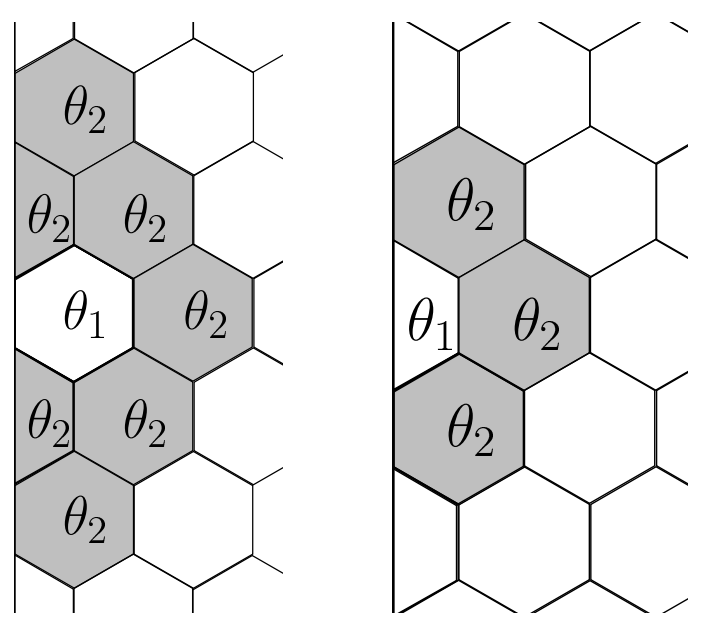

Figure 6: Angles setting the specific configurations for (a) closed and (b) open grains.

if $\theta=45^{\circ}$. After 10 cycles, the expected average value is 1.5 but at the integration point scale it reaches 15 . That is 10 times more than the average value. This is a classical result, showing that strain is strongly localized in bands or at grain boundaries.

Figures 7 and 8 respectively, show the C-45-0 and $\mathrm{O}-$ 45-0 cluster types. In both cases, a soft grain is embedded in a hard surrounding. Twenty realizations are considered for the rest of the aggregate. Each figure recalls the geometrical distribution of the grain orientations, and illustrates three typical cases, by comparing the contour of the Schmid factor and the corresponding field of accumulated viscoplastic shear strain. The far field depends on the arrangement of the randomly chosen orientations. The structure of the localization bands changes for the various realizations. It is worth noting that they do not necessarily follows the softest grains (with the highest values of the Schmid factor). There are two types of strain localization: 

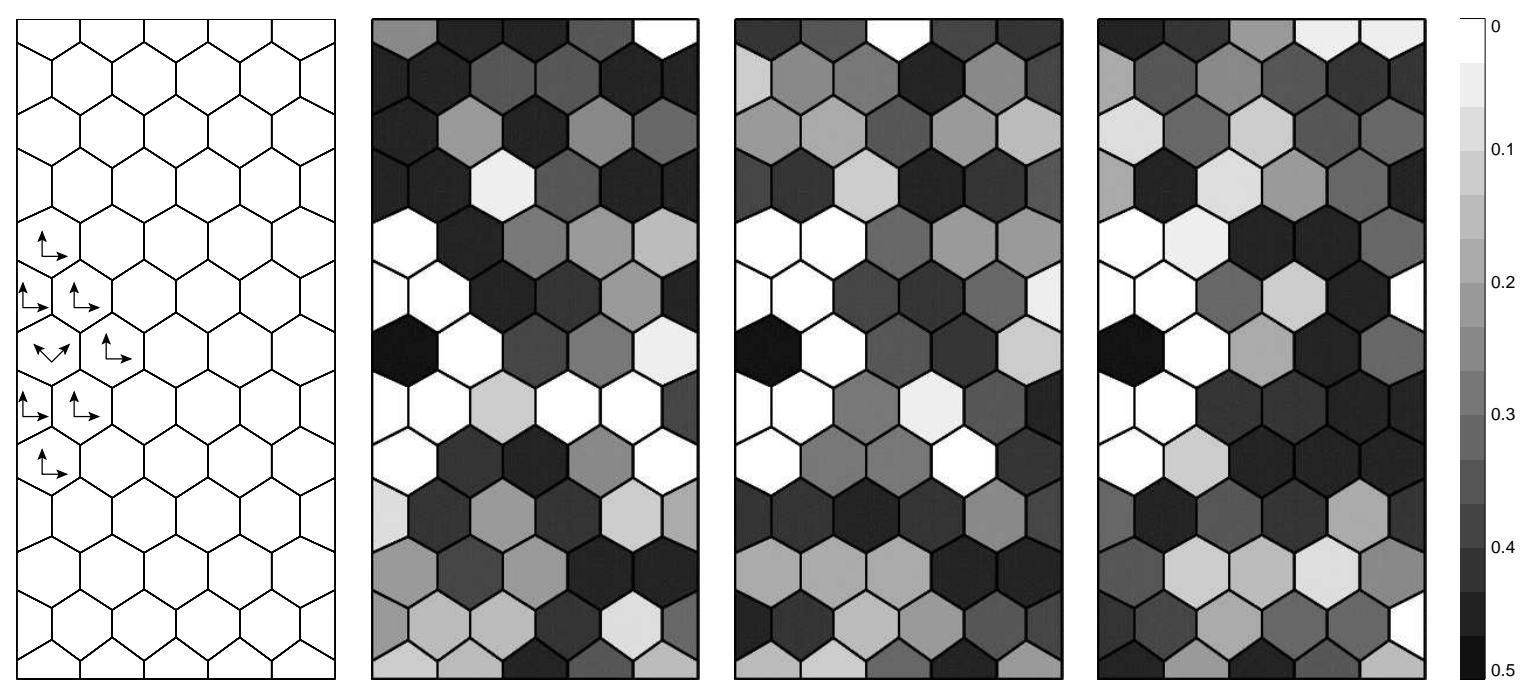

(a)
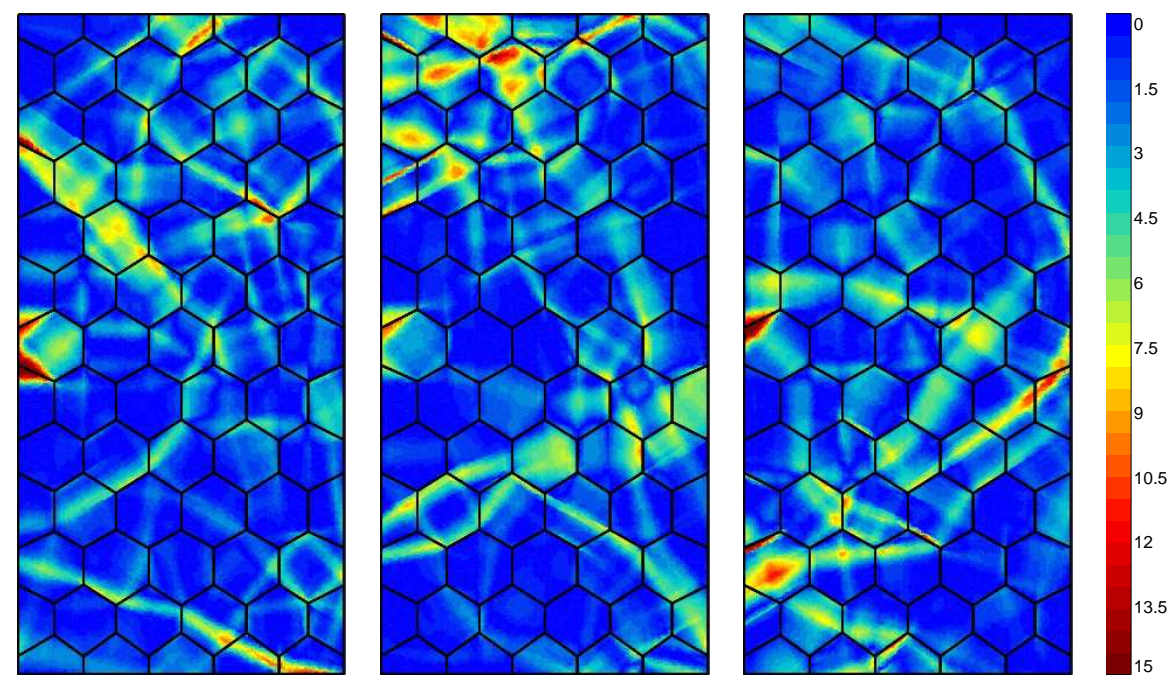

(b)
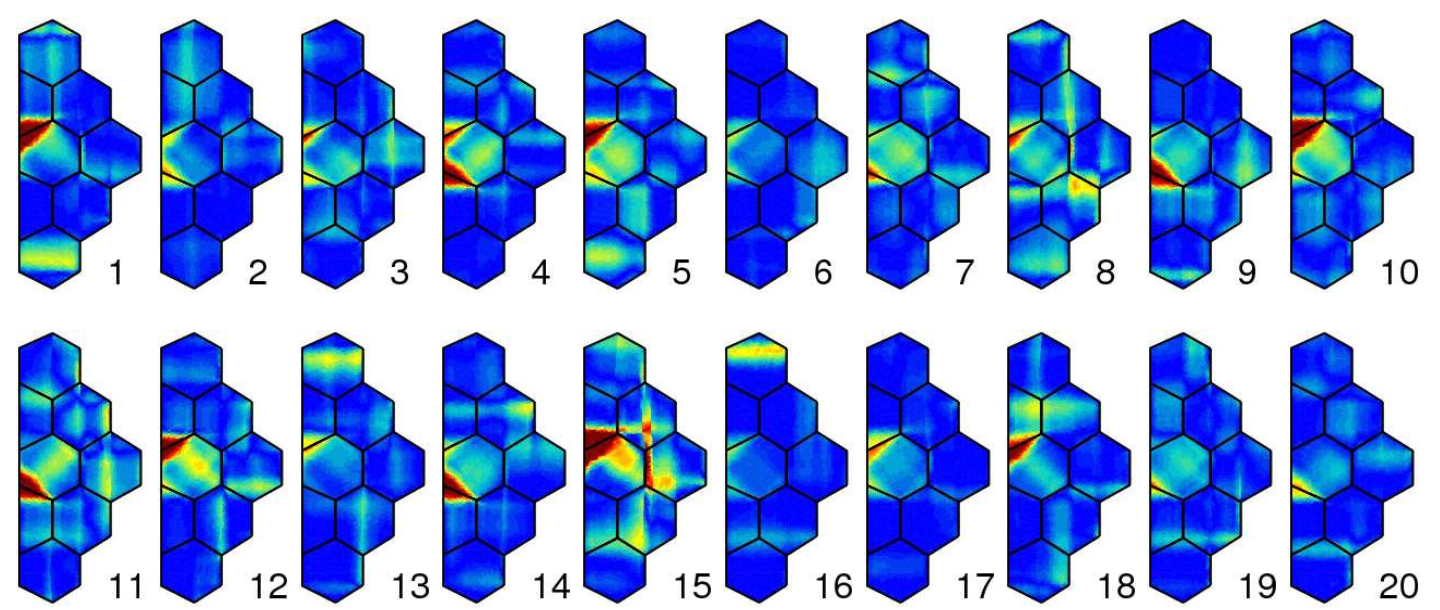

(c)

Figure 7: Cluster C-45-0 : Maps corresponding to different realizations.

Entire maps of (a) Schmid factor and (b) cumulated viscoplastic strain. (c) Local clusters maps of cumulated viscoplastic strain. 

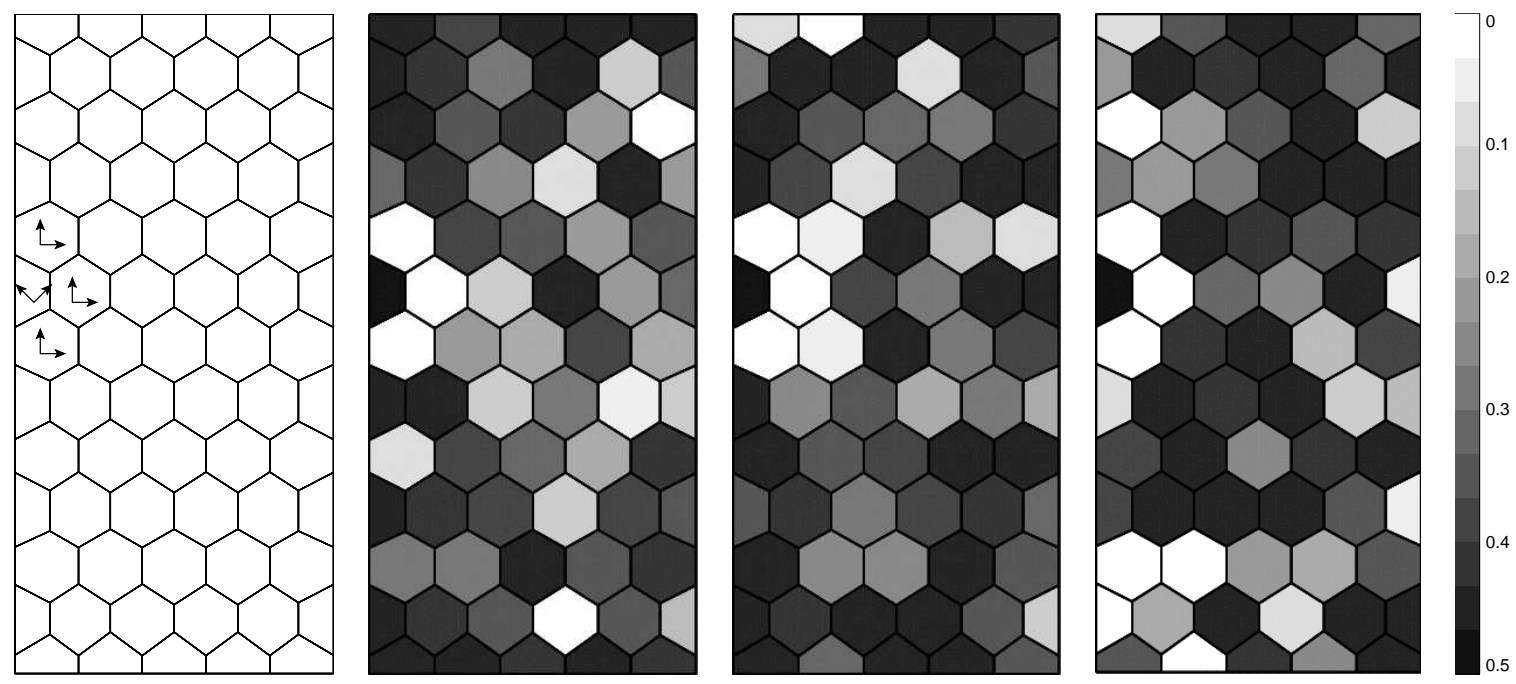

(a)
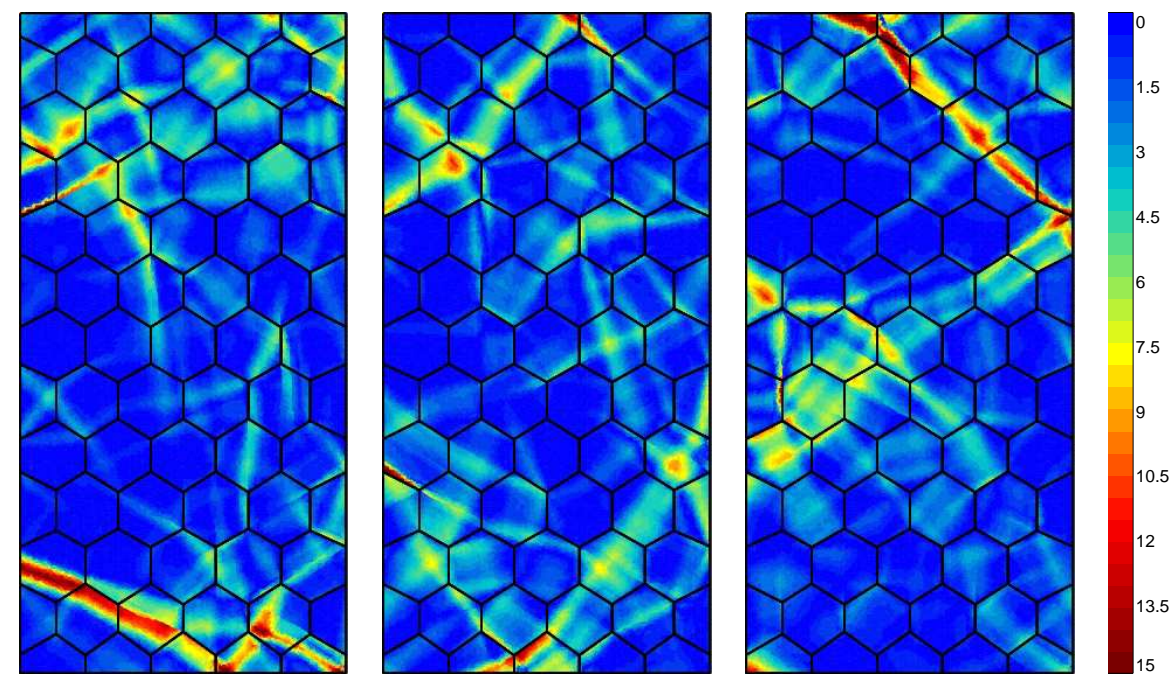

(b)
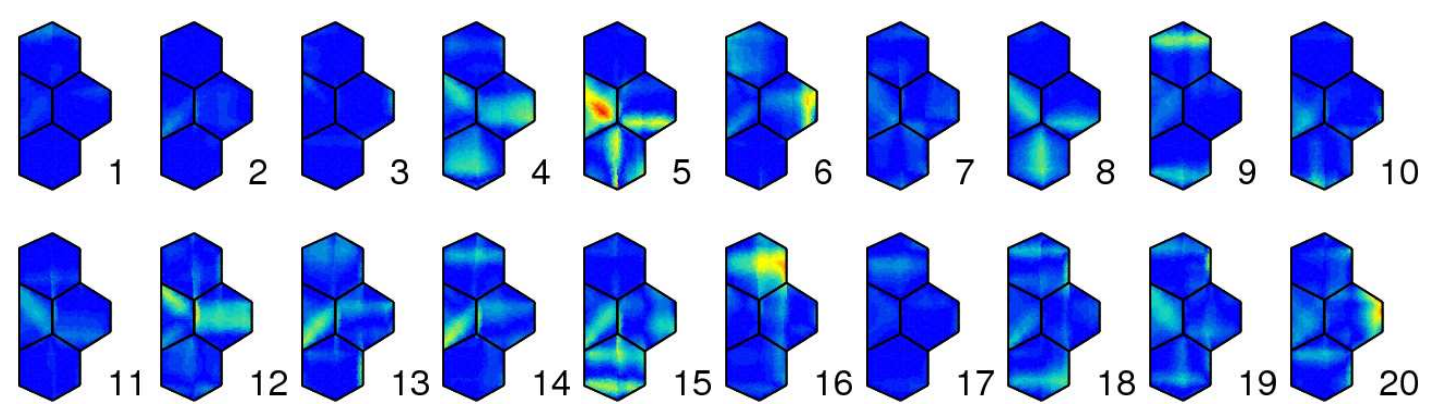

(c)

Figure 8: Cluster O-45-0 : Maps corresponding to different realizations.

Entire maps of (a) Schmid factor and (b) cumulated viscoplastic strain. (c) Local clusters maps of cumulated viscoplastic strain. 
(i) the presence of a chain of soft (or almost soft) grains with a general direction not far from $45^{\circ}$ with respect to the tensile axis promotes localization zones located in the center of the grains: this is "type 1", or primary plasticity, as found in the single crystal.

(ii) the juxtaposition of one soft and one hard grain creates a zone with slip incompatibilities, and a "type 2" band, near the grain boundary, which will be the source of intergranular stresses.

The local fields in the cluster are shown for the 20 computations in Fig. 7c and 8c. They appear to be also dependent on the distribution in the rest of the aggregate. It demonstrates that the response of the central grain depends on the surrounding grains in the cluster, and that the response of the cluster depends also on the rest of the aggregate.

For most of the calculations, the central grain remains highly deformed (type 1 plasticity). Nevertheless, in some cases, the hard neighborhood prevents flow in the central grain (see for instance computations $6,13,16$ for the closed grains in Fig. 7c and 1, 3, 17 for the open grains in Fig. 8c). Type 2 plasticity is also present, as observed in clusters 1 , 4, 14 in Fig. 7c, and 12, 14 in Fig. 8c, where hard grains are crossed by localization bands. Also, it can be seen that open grains present more scatter than the closed ones.

Figures 9 and 10 respectively show the $\mathrm{C}-0-45$ and $\mathrm{O}-$ 0-45 cluster types. The general aspect of the aggregate is the same as the two preceding figures. In the clusters, the grains at $45^{\circ}$ show a localization band, that goes around the hard grain, for the open as well as for the closed case. There is either one or two bands, depending on the orientation of the neighboring grains in the aggregate. As observed for clusters 1 and 12 in Fig. 9c and 3, 9, 18 in Fig. 10c, plasticity may be present in the central grain. For each case, the band seems to emerge due to the strain state in the surrounding grains.

A more quantitative view is provided in Fig. 11 which shows the distribution of the accumulated plastic slip. It is based on 100 different computations for each cluster. The distribution is plotted by using either the values at Gauss points or the averaged values in the grains. It confirms that the strain remains small in hard grains (Fig. 11e and Fig. 11f). Nevertheless, rather large accumulated slip can be also found, especially for the open grains (Fig. 11f). The grains presenting the highest cumulated plastic slip belong to the C-45-0 clusters (Fig. 11a). Introducing open grains instead of closed ones leads to less plastic flow. As observed from Fig. 11b, the shape of the distribution function exhibits a significant number of Gauss points (and of grains) in the smaller class $(0<p<1)$. The average value for open grains is also smaller than the value obtained for closed grains (1.5 versus 2.3 ).

After having considered organized clusters (0-45 or 450 ), the same number of calculations (100 computations in each case) have been performed for the four types of clusters with randomly distributed grains around the core of the cluster. The central grains are either closed $(\mathrm{C}-0$ $\mathrm{R}, \mathrm{C}-45-\mathrm{R})$, or open $(\mathrm{O}-0-\mathrm{R}, \mathrm{O}-45-\mathrm{R})$. The conclusions are similar to those obtained for the four previous computations, but all the trends are less marked. The "perfect 0-45 misorientation" found in the organized cluster magnifies the effects. This is especially true for clusters with the large "closed" grain, where the average value in Fig. $11 \mathrm{~g}$ is larger than the corresponding one in Fig. 11e and the average value in Fig. 11c is smaller than the value in Fig. 11a. Instead of decreasing like for closed grain, the amount of plasticity in soft open grains becomes larger when one considers random neighbors orientation (Fig. 11d) instead of hard one (Fig. 11b).

The results obtained by plotting the average values in the elements confirms the graphs made with Gauss points values. This is summarized in Fig. 12. The ranking of the different curves is the same, the smallest values of the accumulated slip is found in C-0-45 clusters, and the highest values in $\mathrm{C}-45-0$. The next curves in each case correspond to the open clusters (namely $\mathrm{O}-0-45$ and $0-45-0$ ). The four random cases follow the same scheme, so that, from the smallest to the highest amount of slip, one can write

$$
\begin{aligned}
& \mathrm{C}-0-45<\mathrm{O}-0-45<\mathrm{C}-0-\mathrm{R}<\mathrm{O}-0-\mathrm{R}<\ldots \\
& \ldots<\mathrm{O}-45-0<\mathrm{O}-45-\mathrm{R}<\mathrm{C}-45-\mathrm{R}<\mathrm{C}-45-0
\end{aligned}
$$

The following conclusions come from the preceding observations:

(i) the most important parameter governing plastic slip at the local scale remains the crystallographic orientation of the central grain $\theta_{1}$;

(ii) closed grains are able to impose their proper behavior, so that they are approaching the response of the single crystal. On the contrary, open grains are prone to cluster effect;

(iii) setting the orientation of the first neighbors of the surrounding grains to a prescribed value is not sufficient to unify its response for the various aggregates, nevertheless, this tends to magnify the cluster effect.

After the orientation of the central grain, the critical parameter is the geometry of the grain. The orientation of the external grains in the cluster is also a significant parameter.

\subsubsection{Local multiaxiality}

Figure 13 presents the local stress multiaxiality for the central grain of each cluster during the whole simulation, e.g. the 10 cycles. $\sigma_{22}$ is the normal component in the tensile direction. $\sigma_{11}$ is the normal component in the direction of the free surface, which is equal to zero at the surface of the aggregate. It is worth noting that the value of $\sigma_{11}$ increases rapidly inside the grain due to grain-grain incompatibilities. It was decided to plot the average of these stress components in each grain, and it can be observed that the average in the grain is far from zero. The purpose of the graph is to underline the difference between the 

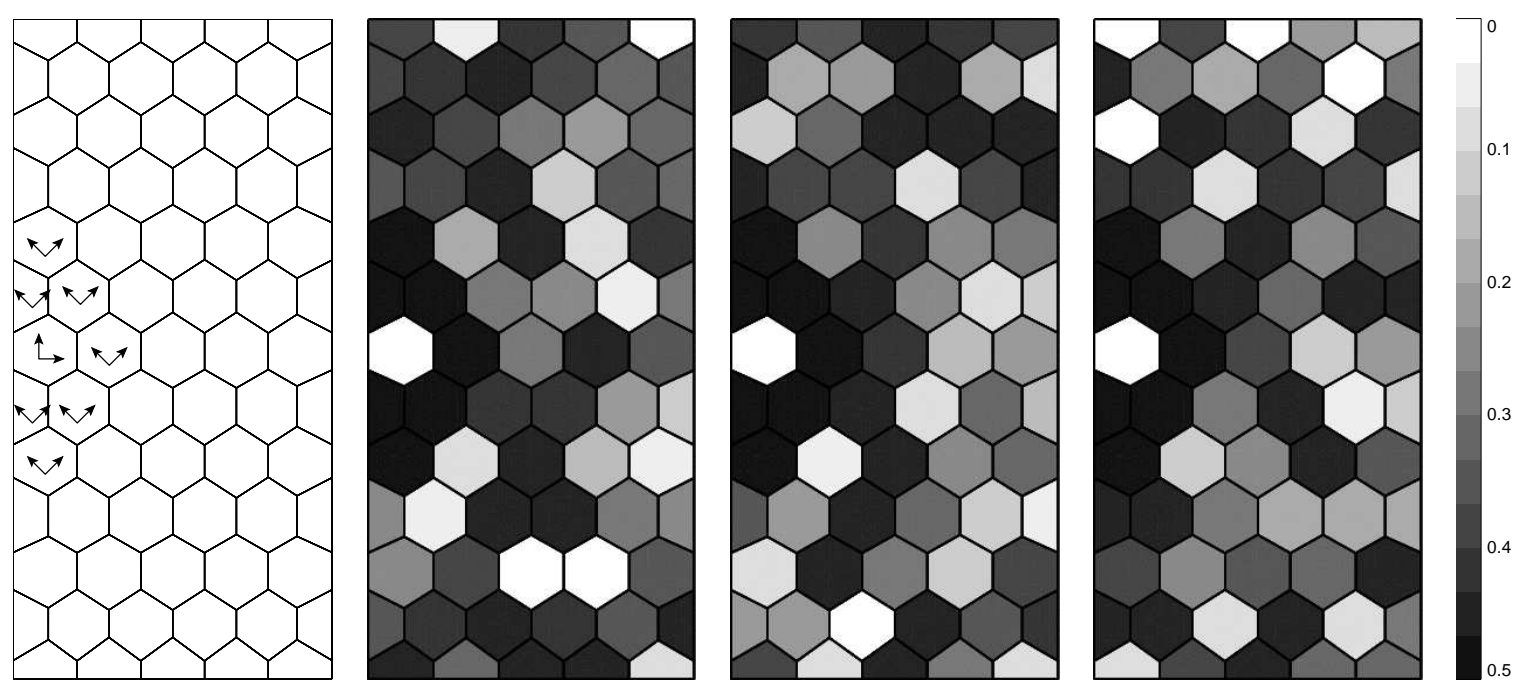

(a)
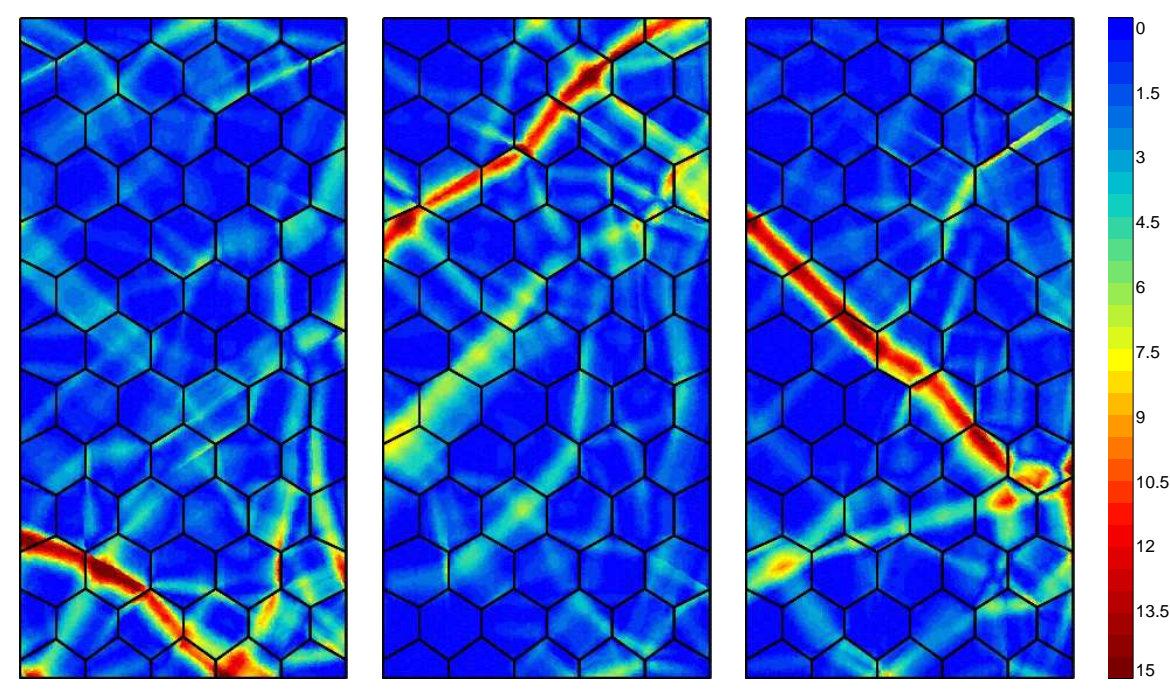

(b)
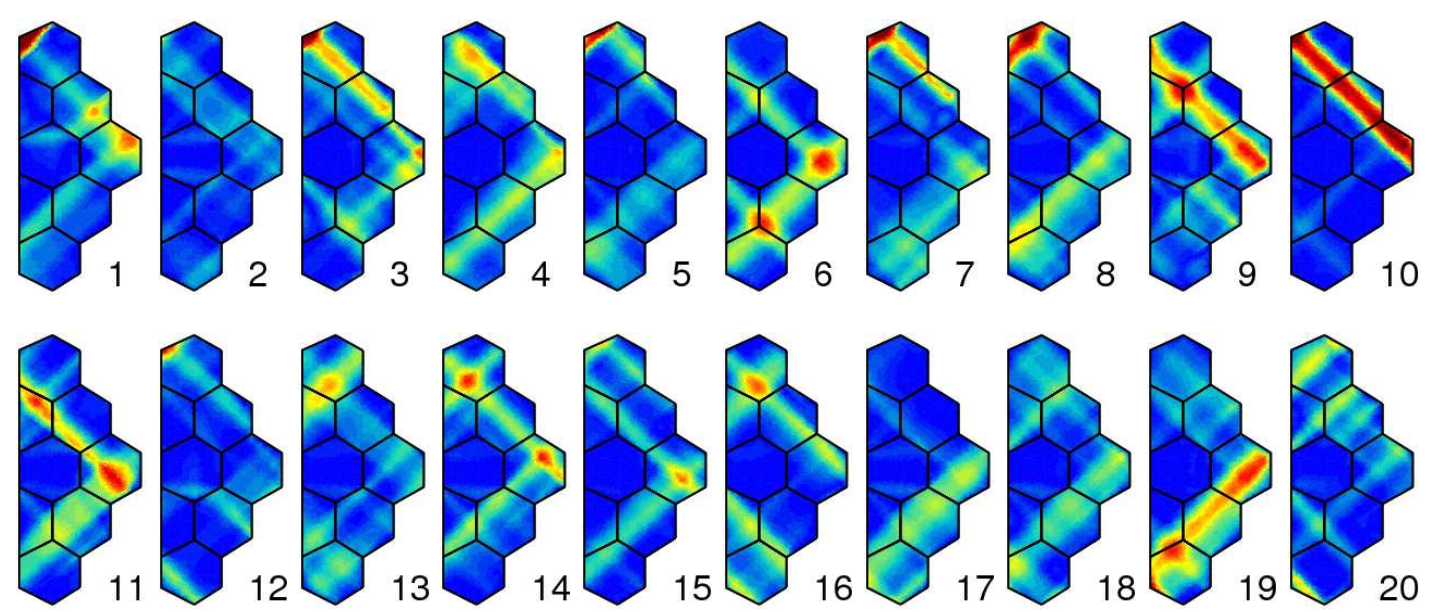

(c)

Figure 9: Cluster C-0-45 : Maps corresponding to different realizations.

Entire maps of (a) Schmid factor and (b) cumulated viscoplastic strain. (c) Local clusters maps of cumulated viscoplastic strain. 

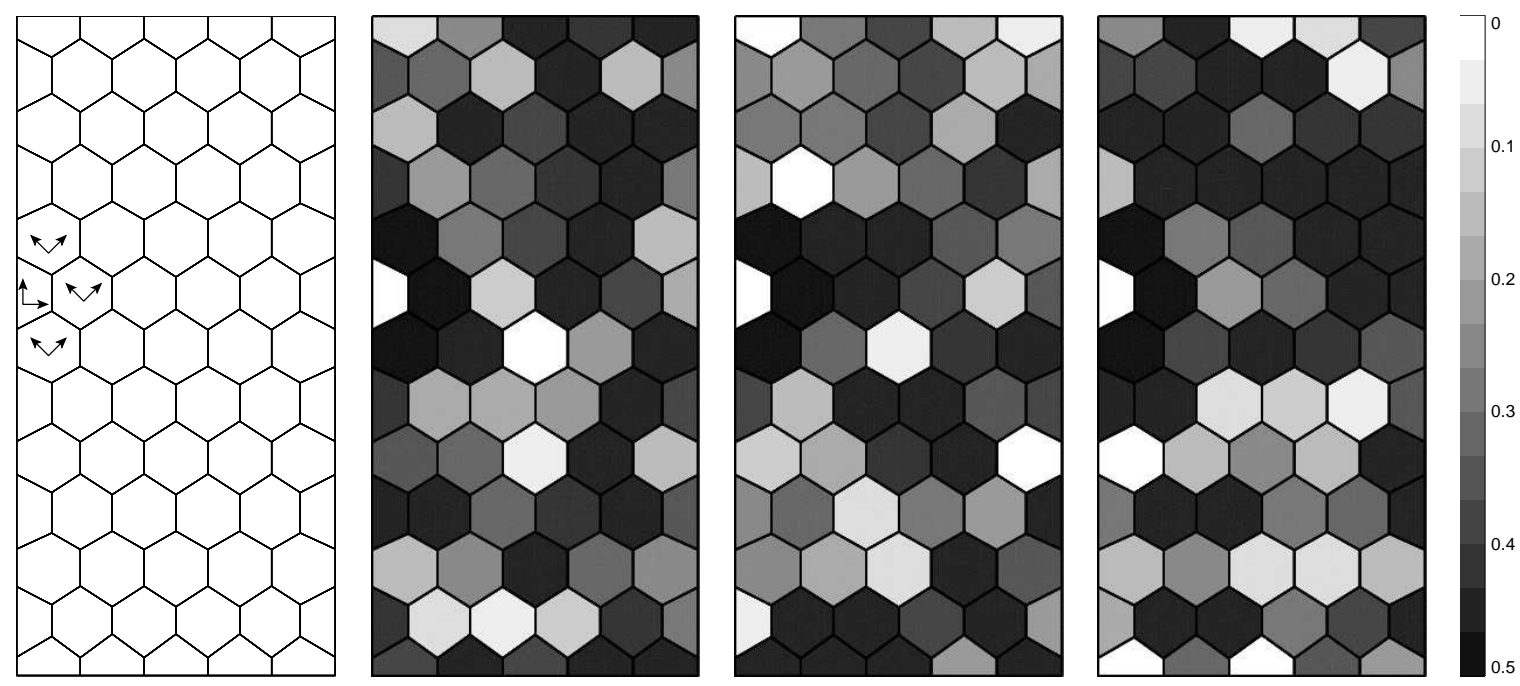

(a)
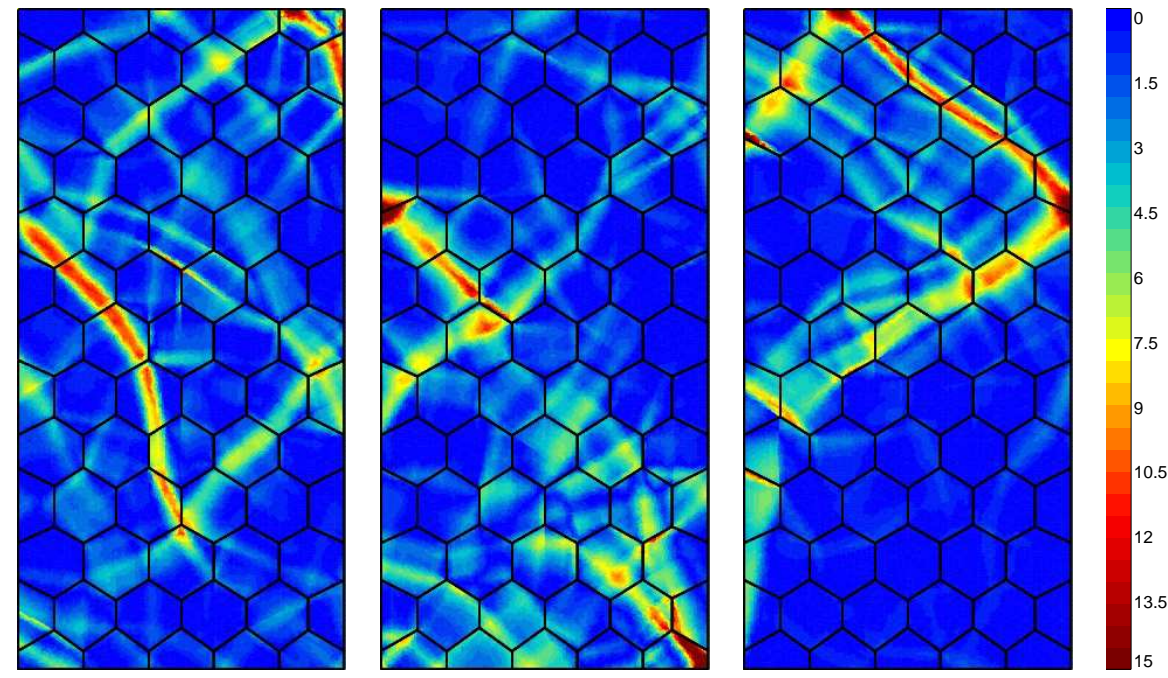

(b)
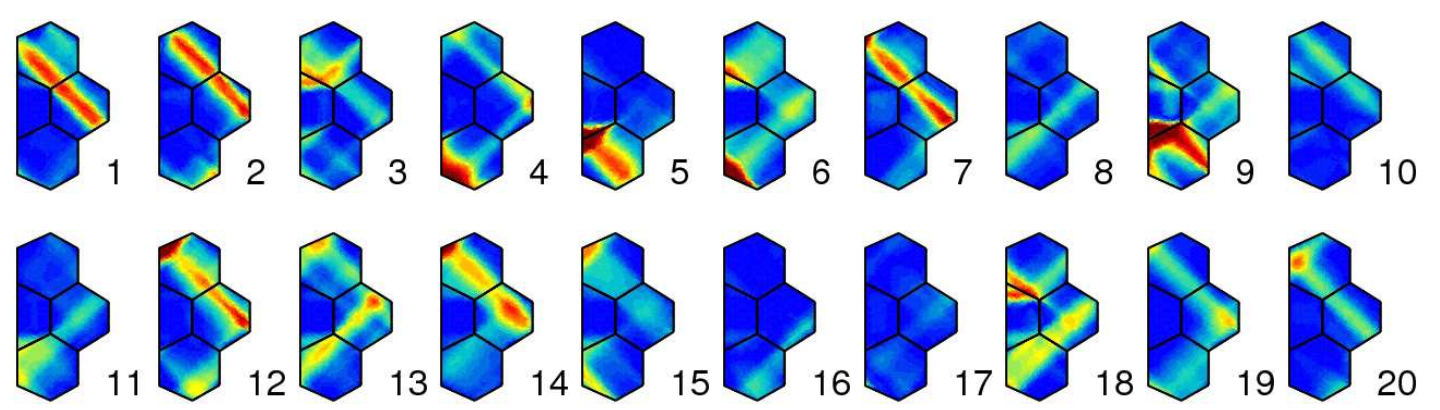

(c)

Figure 10: Cluster $\mathbf{O}-\mathbf{0 - 4 5}$ : Maps corresponding to different realizations

Entire maps of (a) Schmid factor and (b) cumulated viscoplastic strain. (c) Local clusters maps of cumulated viscoplastic strain. 


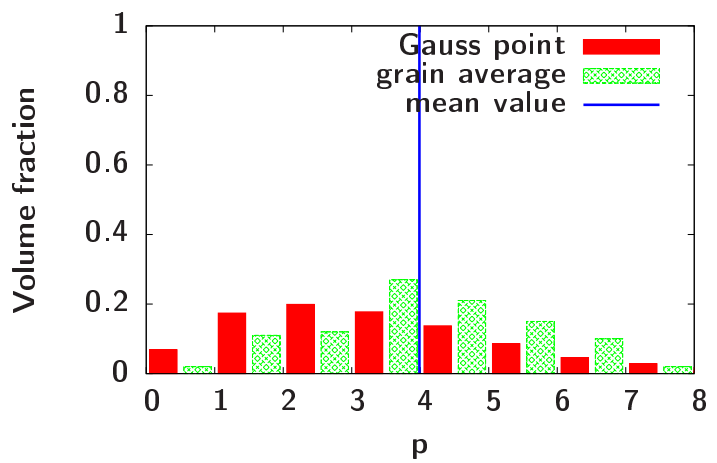

(a) $\mathrm{C}-45-0$

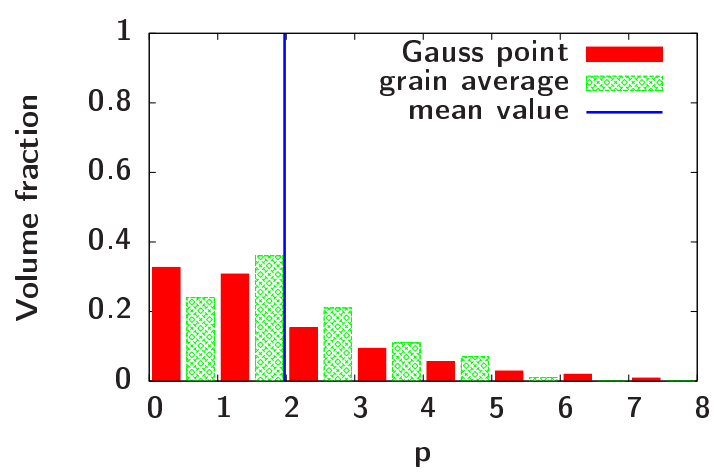

(b) $\mathrm{O}-45-0$

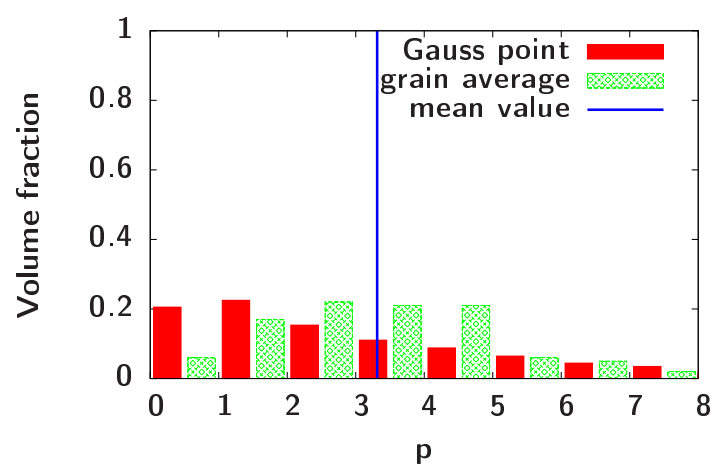

(c) $\mathrm{C}-45-\mathrm{R}$

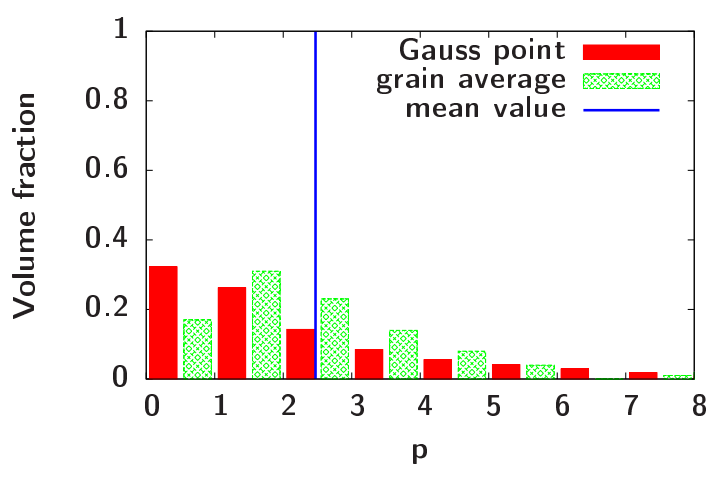

(d) $\mathrm{O}-45-\mathrm{R}$

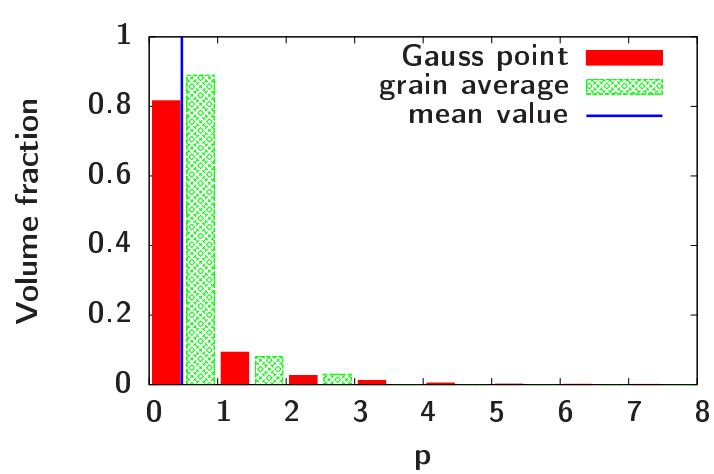

(e) $\mathrm{C}-0-45$

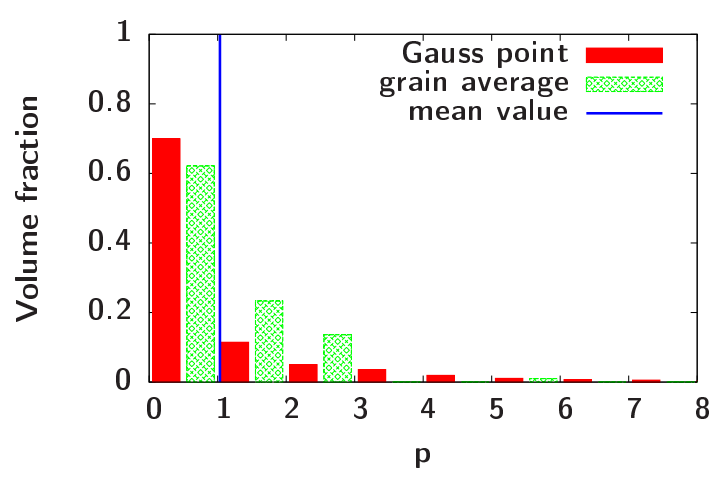

(f) $\mathrm{O}-0-45$

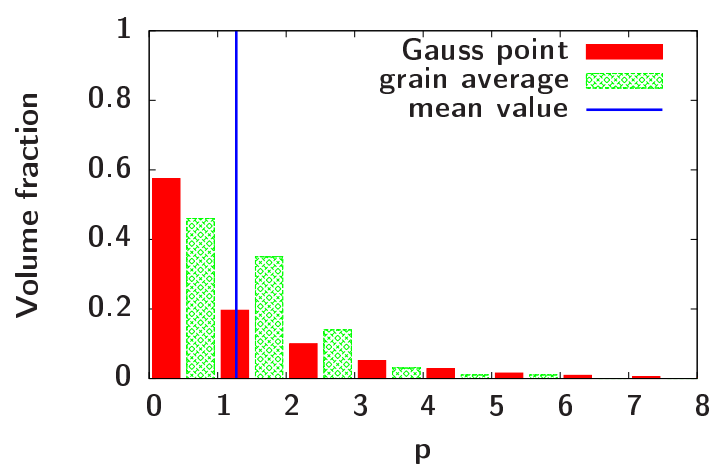

(g) $\mathrm{C}-0-\mathrm{R}$

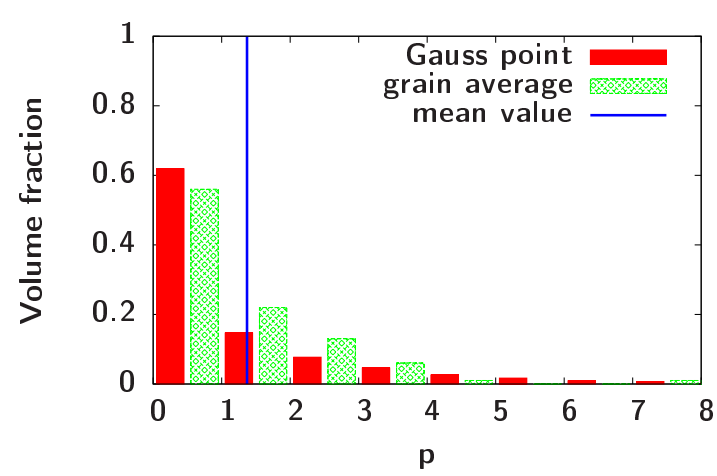

(h) $\mathrm{O}-0-\mathrm{R}$

Figure 11: Comparison of the distribution of the cumulated plastic strain in the central grain for various clusters. The values are taken from the FE field (Gauss points) or from the average in each grain. The central grain is either "open" (O) or "closed" (C). The first number shows the orientation of the central grain, either soft (45) or hard (0). The last item defines the orientation of the neighboring grains in the cluster, soft (45), hard (0) or random (R). 


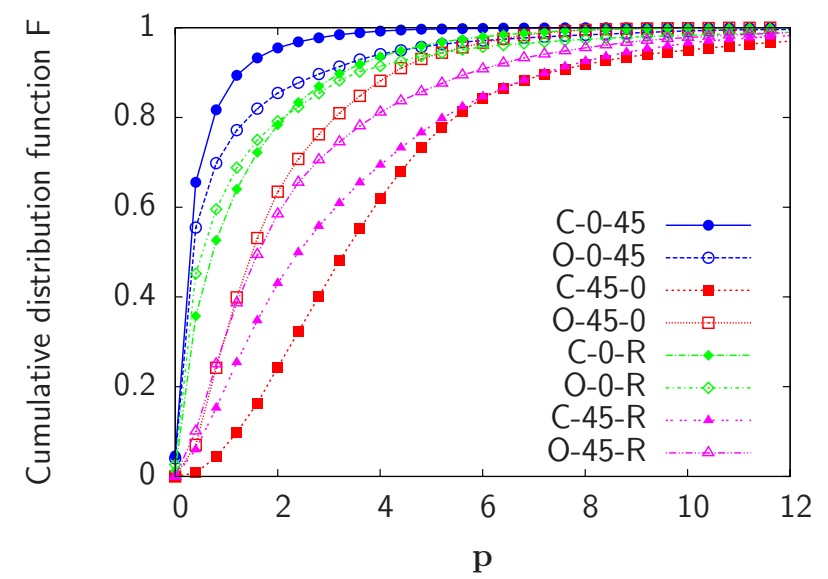

(a)

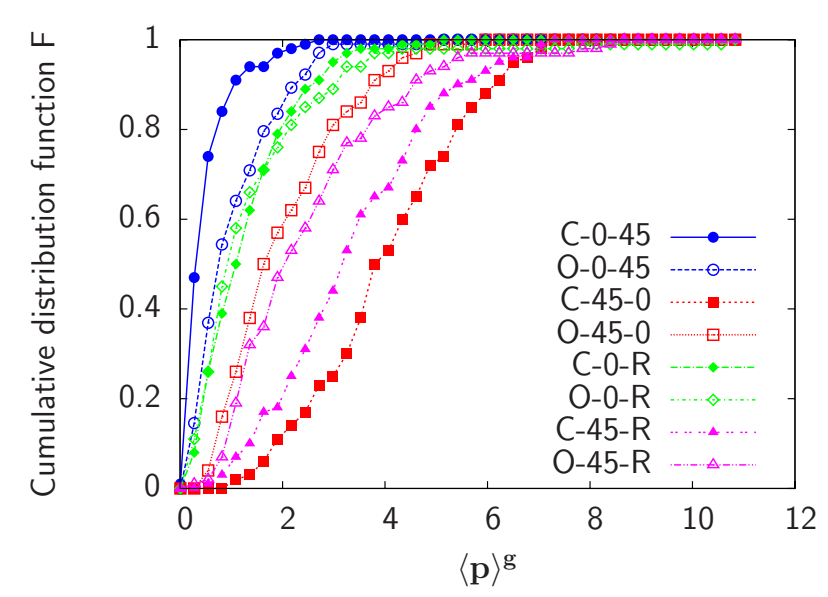

(b)

Figure 12: Cumulative distribution functions of cumulated viscoplastic strain for different types of clusters based on central grain (a) Gauss points values and (b) grain averages.

two types of aggregate (soft grain surrounded by hard material or hard grain surrounded by soft material). Twenty curves corresponding to various realizations are shown for each of the eight cases. For all of them, the general aspect is a parallelogram.

The main difference between the results for the different types of clusters comes again from the orientation of the central grain $\theta_{1}$. Indeed, for soft central grains (C-45-0 in Fig. 13a, $\mathrm{O}-45-0$ in Fig. $13 \mathrm{~b}, \mathrm{C}-45-\mathrm{R}$ in Fig. $13 \mathrm{c}$, $\mathrm{O}-$ 45-R in Fig. 13d), curves show two vertical lines since the 11 component does not change during elastic part of the strain path. The cycles are closed by two lines whose slope is equal to 1. During plastic flow, the increments of both components are equal, and a positive value for $\sigma_{22}$ generates the same amount of tensile stress on $\sigma_{11}$. This is verified for the "ideal" cases C-45-0 and O-45-0, but also for the clusters with randomly generated external grain orientations ( $\mathrm{C}-45-\mathrm{R}$ and $\mathrm{O}-45-\mathrm{R})$. The open/closed character of the central grain is not sensitive.

Such a slope is easily recovered by a simplistic model, formed of six generalized plane strain finite elements (see Fig.14 which emulates the local behavior of the clusters. The grain 0 (central grain of the cluster) is surrounded by five elements representing the first layer of grains. All the edges remain parallel to their initial direction so that the stress tensors are uniform in each element. A pressure is applied on the top and bottom boundaries of the mesh. One element (number 0) has an elastoplastic behavior, the other five remain elastic. Assuming that grains 1 and 5 are strong enough to avoid plastic flow, their transverse strain remains negligible. In this case, the strain and stress tensors of grain 0 can be expressed as

$$
\underset{\sim}{\varepsilon}=\left(\begin{array}{ccc}
\approx 0 & 0 & 0 \\
& \varepsilon & 0 \\
& & 0
\end{array}\right) \Rightarrow \underset{\sim}{\sigma}=\left(\begin{array}{ccc}
\sigma_{1} & 0 & 0 \\
& \sigma_{2} & 0 \\
& & \approx \sigma_{1}
\end{array}\right)
$$

The yield function becomes $\sigma_{1}-\sigma_{2}=R_{0}$ and a slope of
1 is found in each $*-45-*$ configuration. This slope of 1 is found for any type of plasticity (crystal plasticity or von Mises), and any value of Poisson's ratio (the elasticity is assumed to be isotropic and uniform in the six elements).

For hard central grains $(\mathrm{C}-0-45$ in Fig. $13 \mathrm{e}$ O-0-45 in Fig. 13f. $\mathrm{C}-0-\mathrm{R}$ in Fig. 13g, $\mathrm{O}-0-\mathrm{R}$ in Fig. 13h), there is much more scatter in the various responses. It can be observed that the ratio $\sigma_{22} / \sigma_{11}$ is negative. A slope of -1 is marked as a reference on each plot. Unlike the case of the soft embedded grain, the difference between the open grains and the closed grains is noticeable. The component $\sigma_{11}$ remains low for open grains (the free surface effect is seen in the whole grain) so that the slope $\sigma_{22} / \sigma_{11}$ has a large value. It was not the case for the $\mathrm{O}-45-0$ and the $\mathrm{O}-45-\mathrm{R}$ cases, where the central grain is weak, and the stress transfer is rapidly imposed by the external grains.

This last observation can be explained by means of the simple six element model (Fig, 14). Assuming that grain 0 remains elastic and the other five become plastic, the slope is exactly -1 for crystal plasticity or von Mises plasticity model. The value of the slope depends on Poisson's ratio. Considering crystal plasticity in the surrounding grains, the strain and stress tensors in the central grain are close to

$$
\underset{\sim}{\varepsilon}=\left(\begin{array}{ccc}
-\varepsilon & 0 & 0 \\
& \varepsilon & 0 \\
& & 0
\end{array}\right) \Rightarrow \underset{\sim}{\sigma}=\left(\begin{array}{ccc}
-\sigma & 0 & 0 \\
& \sigma & 0 \\
& & 0
\end{array}\right)
$$

So $\sigma_{11}=-\sigma_{22}$ and a negative slope of -1 is found.

\section{Fatigue life prediction}

The FE analysis is now performed for different loading amplitudes $( \pm 0.3 \%, \pm 0.5 \%, \pm 1 \%$ and $\pm 2 \%)$. The three criteria presented in section 2.2 are evaluated by postprocessing of the 10th cycle of the problem (stabilized cycle). As earlier, the study is made on random aggregates and clusters. 


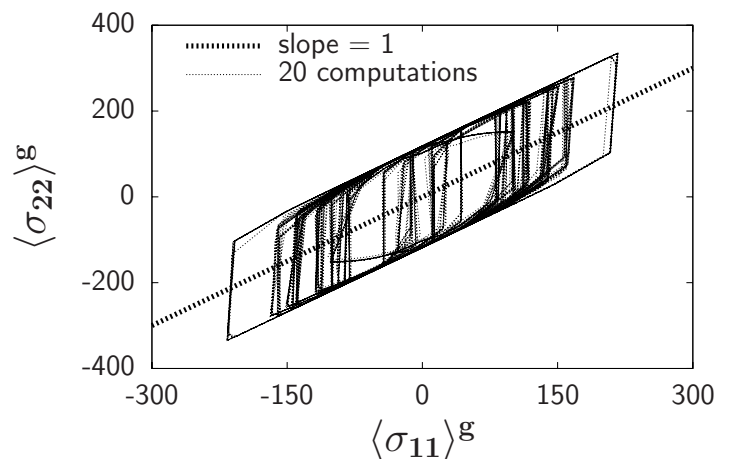

(a) $\mathrm{C}-45-0$

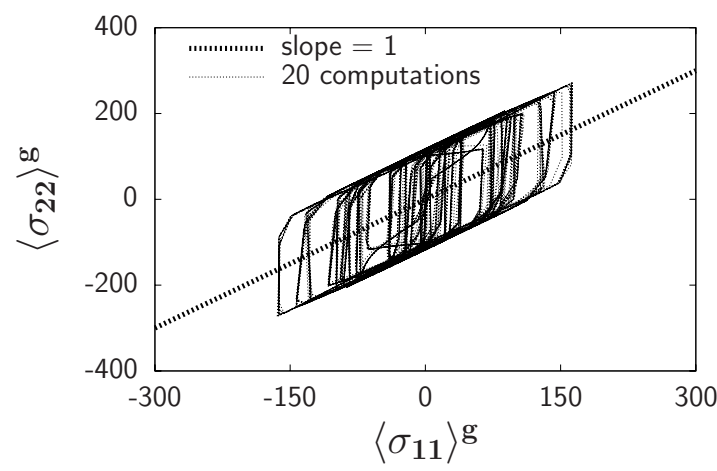

(b) $\mathrm{O}-45-0$

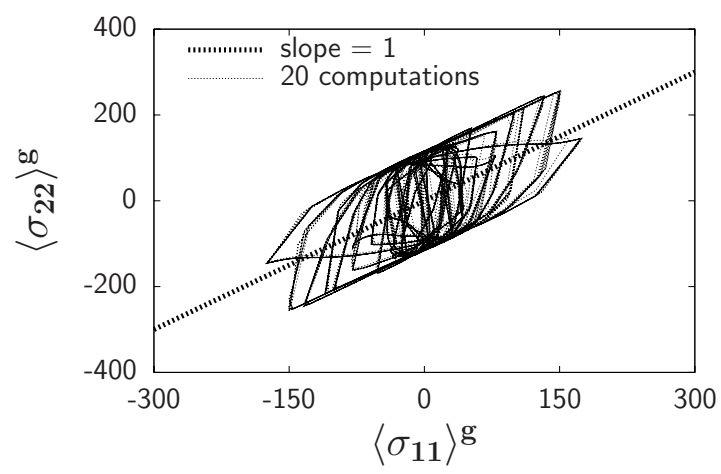

(c) $\mathrm{C}-45-\mathrm{R}$

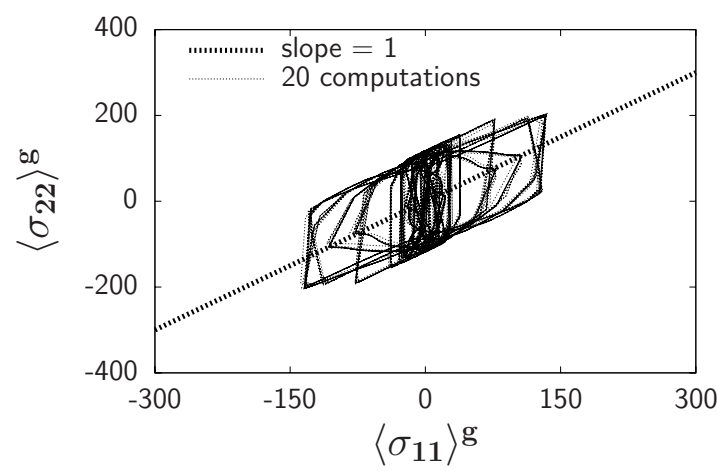

(d) $\mathrm{O}-45-\mathrm{R}$

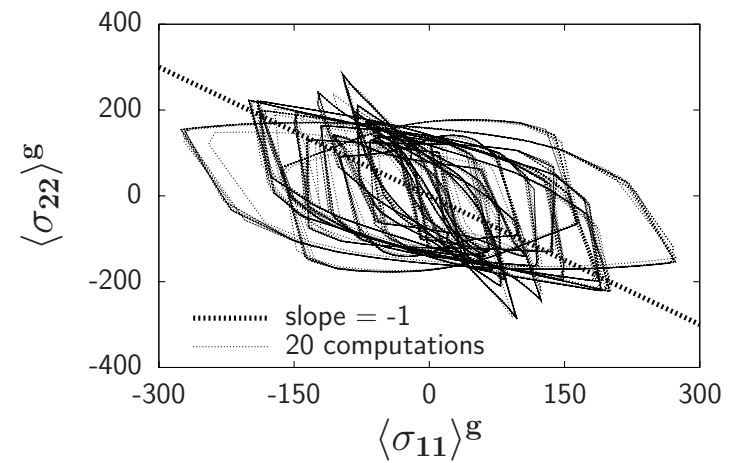

(e) $\mathrm{C}-0-45$

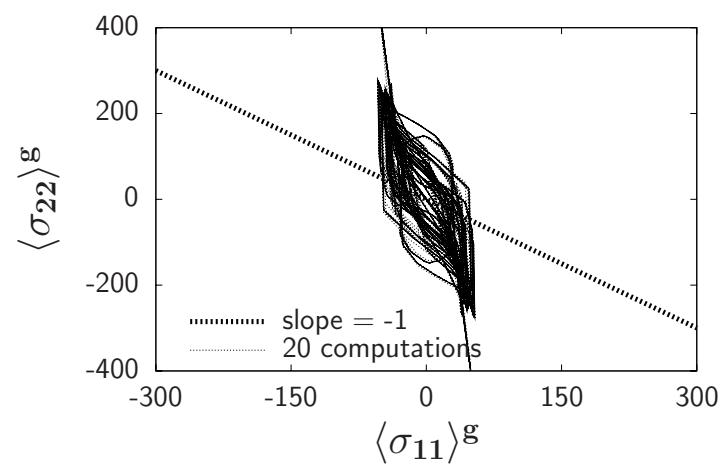

(f) $\mathrm{O}-0-45$

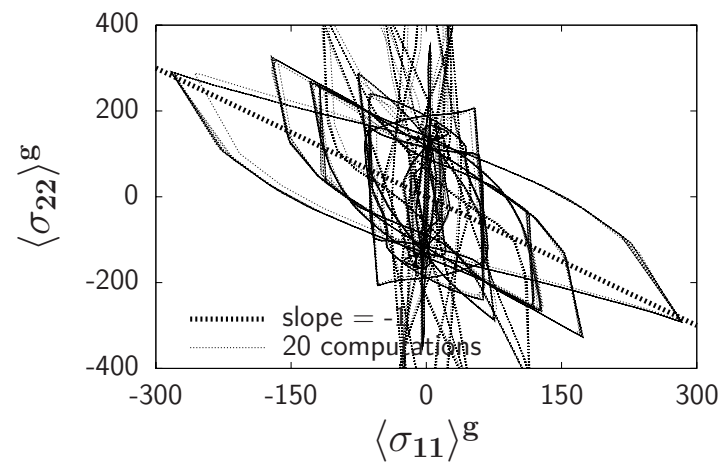

(g) $\mathrm{C}-0-\mathrm{R}$

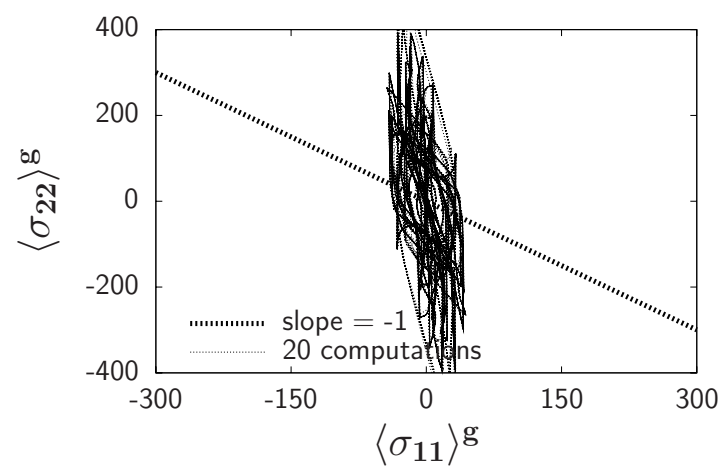

(h) $\mathrm{O}-0-\mathrm{R}$

Figure 13: $\sigma_{22} / \sigma_{11}$ multiaxiality inside the central grain of different clusters. Pictures related to one soft grain inside hard grains are in the left hand column, those with one hard grain inside soft grains are on the right. 


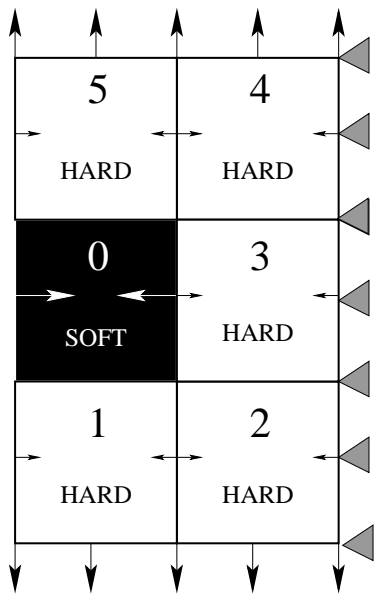

(a)

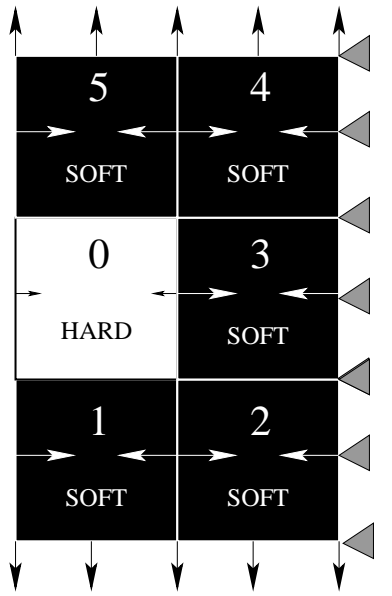

(b)

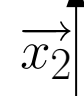

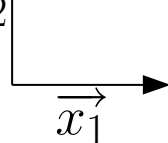

Figure 14: Simple model explaining different states of stress multiaxiality for a tensile loading in direction $x_{2}$. (a) Tensile $\sigma_{11}$ in the soft element, (b) compressive $\sigma_{11}$ in the hard element.

\subsection{Fatigue on random aggregates}

Fifty computations (i.e. total of 3550 grains) were performed for each loading amplitude. Figure 15 shows the values of the criteria depending on grain orientation at each loading level.

For the three criteria, the influence of the grain orientation is more important at low strain range than at large strain range. This is related to the strain localization that is more active at lower strain amplitudes. The Dang Van equivalent stress [37] does not present large variations (Fig. 15a 15d). Nevertheless, since the exponent of the power law in stress dependent fatigue models is high, the effect on life prediction is comparable for this approach and for the strain dependent models (Socie-Fatemi 36], Fig. $15 \mathrm{e} 15 \mathrm{~h}$ or cumulated slip, Fig. 15i 15l). For all criteria, grains with slip planes at $45^{\circ}$ with respect to the tensile axis are the most critical. The other remarkable effect concerns the location of crack initiation. Critical values of both $\sigma_{D V}$ and $\gamma_{S F}$ are higher for the core grains. They do not predict surface crack initiation. In this case they can then hardly be used as a micromechanichal model to represent local physical phenomena. On the other hand, the variable $p$ is maximum around $45^{\circ}$ for the surface grains open or closed - and is a good candidate.

\subsection{Fatigue on clusters}

For each loading level, 20 computations were performed for each clusters. Only $*-0-45$ and $*-45-0$ configurations are shown in order to have a clear view of the results. Figure 16 reports the cumulative distributions of the fatigue criteria values at the Gauss point scale inside the central grain of the clusters. Each criterion produces an important scatter at the intragranular level, as shown by the values at the Gauss points. This scatter is present even inside the central grain of the same cluster. The criteria distributions present higher values for $\mathrm{C}-45-0$ clusters whereas C $-0-45$ clusters have the lowest. This confirms that the $\mathrm{C}-45-0$ configuration is the most critical and conversely that $\mathrm{C}-0-45$ is the safer. At lower loading levels, the results for open or closed clusters are quite similar while the results for soft and hard clusters are rather different (see Fig. 16a, 16e and 16il). But when the load becomes larger, both trends are reversed. Closed clusters become more critical than open ones and the gap between soft and hard grains decreases (see Fig. 16d, 16h and 161.

\section{Conclusions}

The main purpose of the study was to build a simplified framework, representative of the local behavior of polycrystals, in order to analyse the stress redistribution and the local multiaxiality under one-dimensional cyclic loading. What is expected is a new view toward physically consistent critical variables, to be introduced in a new class of multiscale fatigue damage models.

It has been shown that crystal orientation is not the only critical parameter that characterizes grain behavior. The other points of interest are grain location (surface, core) and the neighboring grains. Local failure must then be considered as the result of a cluster effect, for which the most critical configurations must be determined.

The other original point is the analysis of the local multiaxial stress states. Under a global one-dimensional tension loading, soft grains embedded in a hard surrounding are submitted to a high hydrostatic stress. Considering the same macroscopic loading, hard grains embedded in a soft surrounding experience shear stress states. This will have important consequences for the local crack initiation model.

The evaluation of various macroscopic models tends to demonstrate that they cannot be just fed by variables defined on a microscale. The only acceptable result (initiation at the surface of the specimen) is obtained with the accumulated plastic slip. In fact, a criterion taking into account the amount of extrusion will be defined in the future. Authors' opinion is that a model describing macroscopic initiation must combine accumulated slip for micro initiation and a combination of the maximum principal stress and shear stress for the micro-propagation. This conjecture will be verified in a forthcoming study. The investigations will use a finer mesh, and a 3D geometry. Twelve octahedral slip systems will be considered. The results will be compared with experimental observations on 316L stainless steel.

\section{References}

[1] K. J. Miller, The behaviour of short fatigue cracks and their initiation. Part II-A General summary, Fatigue and Fracture of Engng Mat and Struct 10 (1987) 93-113. 


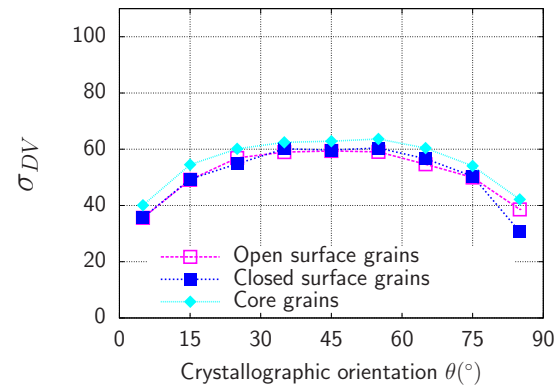

(a) $\sigma_{D V}$ for $\Delta E / 2=0.3 \%$

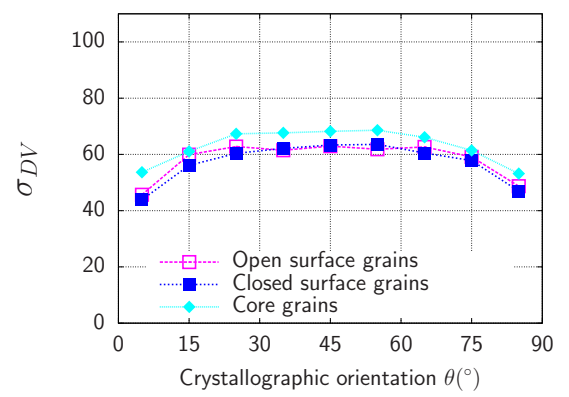

(b) $\sigma_{D V}$ for $\Delta E / 2=0.5 \%$

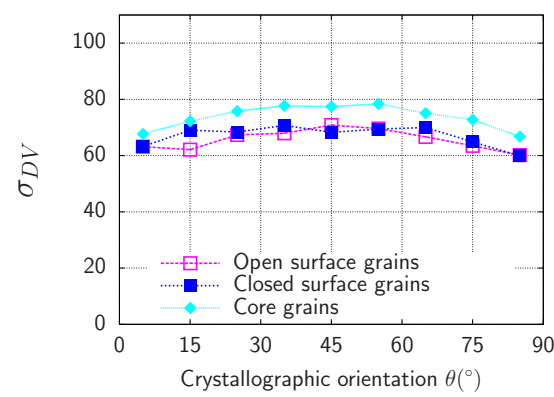

(c) $\sigma_{D V}$ for $\Delta E / 2=1.0 \%$

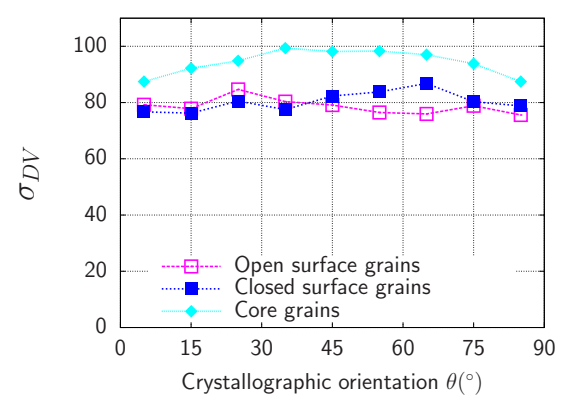

(d) $\sigma_{D V}$ for $\Delta E / 2=2.0 \%$

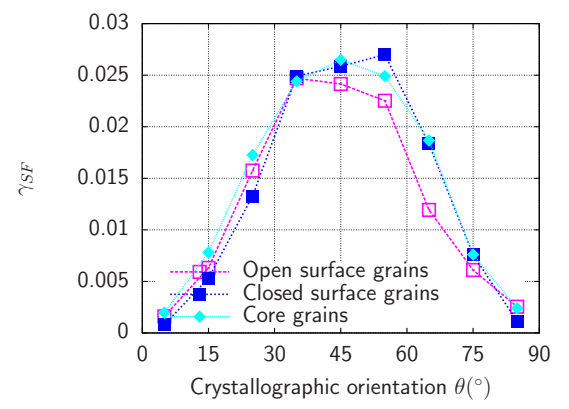

(e) $\gamma_{S F}$ for $\Delta E / 2=0.3 \%$

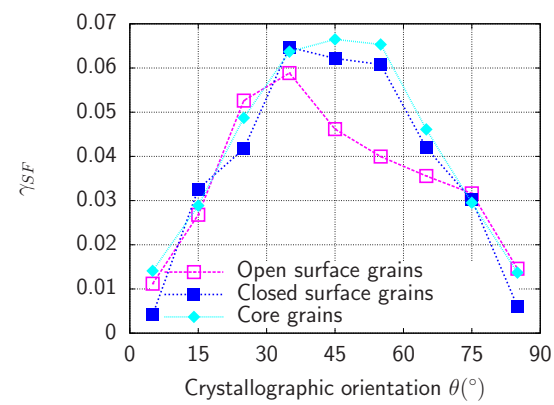

(f) $\gamma_{S F}$ for $\Delta E / 2=0.5 \%$

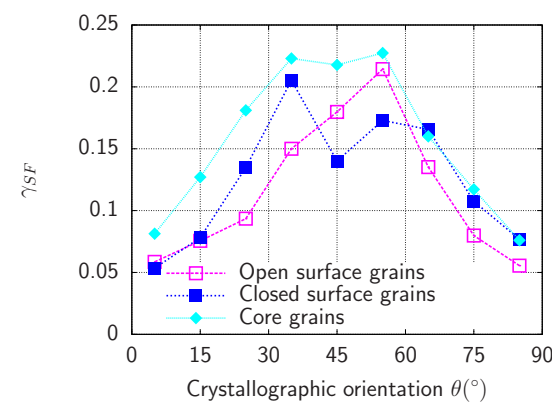

(g) $\gamma_{S F}$ for $\Delta E / 2=1.0 \%$

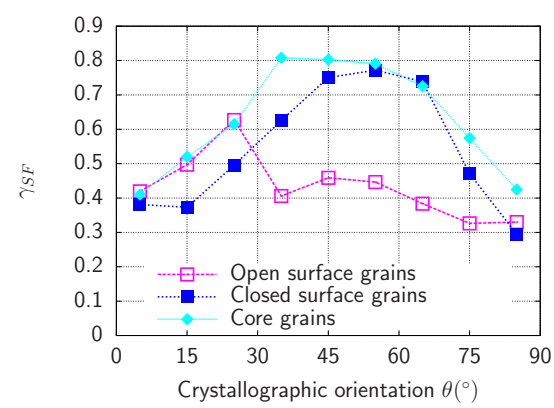

(h) $\gamma_{S F}$ for $\Delta E / 2=2.0 \%$

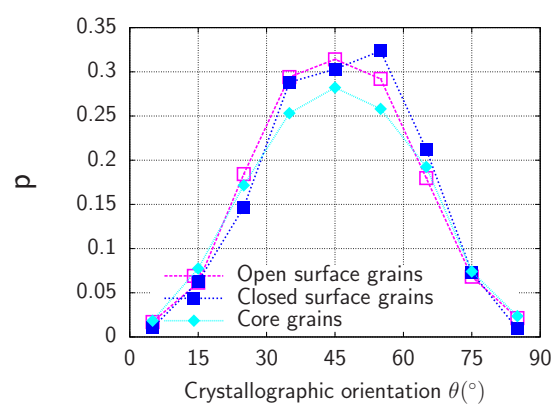

(i) $p$ for $\Delta E / 2=0.3 \%$

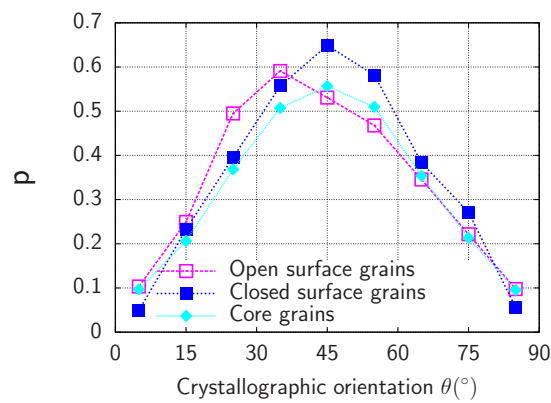

(j) $p$ for $\Delta E / 2=0.5 \%$

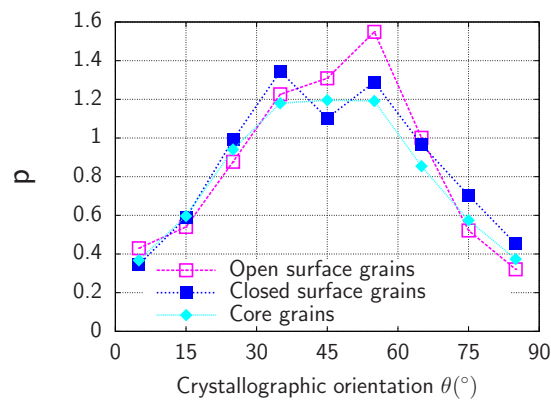

(k) $p$ for $\Delta E / 2=1.0 \%$

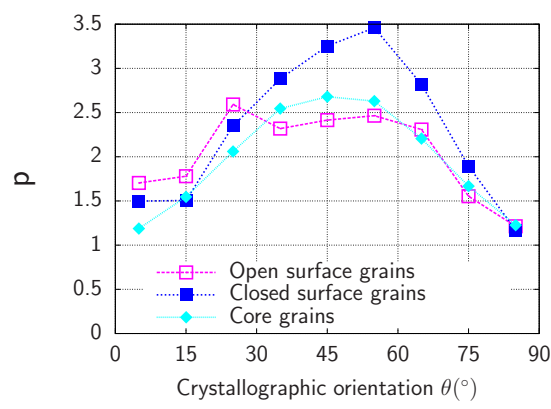

(l) $p$ for $\Delta E / 2=2.0 \%$

Figure 15: Values of different fatigue criteria related to crystallographic orientation for different loading amplitudes (grain average values). 


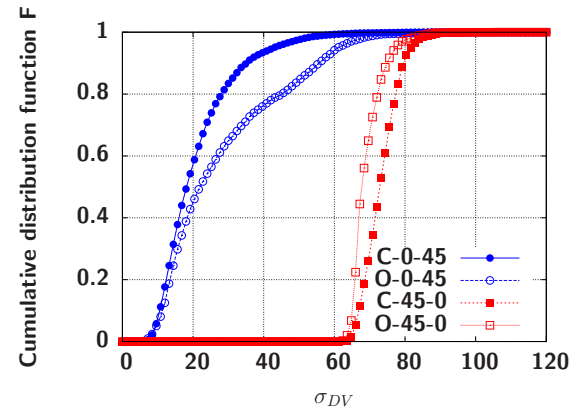

(a) $\Delta E / 2=0.3 \%$

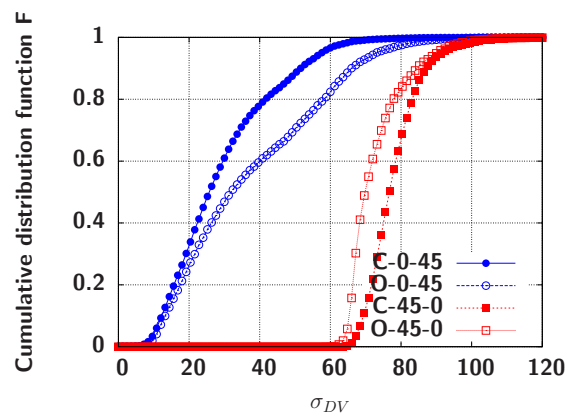

(b) $\Delta E / 2=0.5 \%$

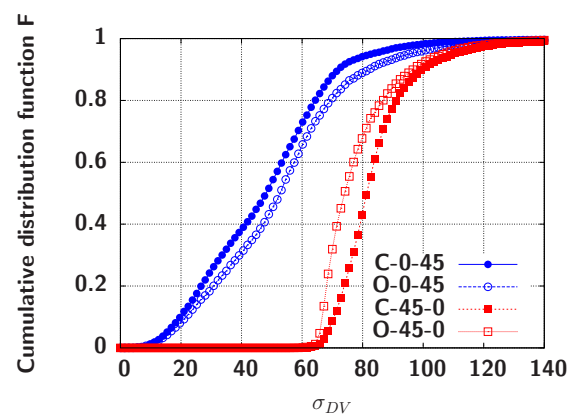

(c) $\Delta E / 2=1.0 \%$

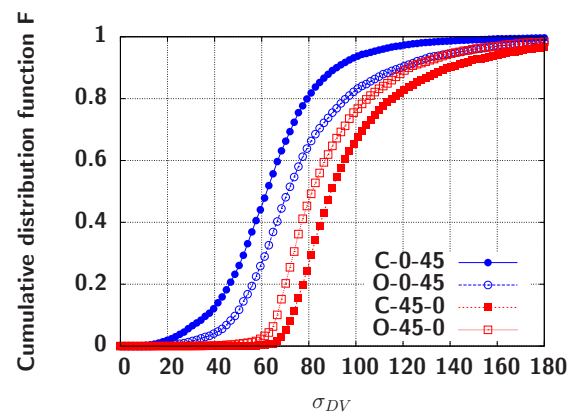

(d) $\Delta E / 2=2.0 \%$

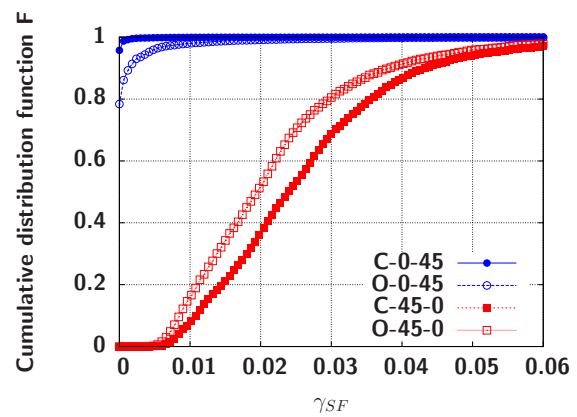

(e) $\Delta E / 2=0.3 \%$

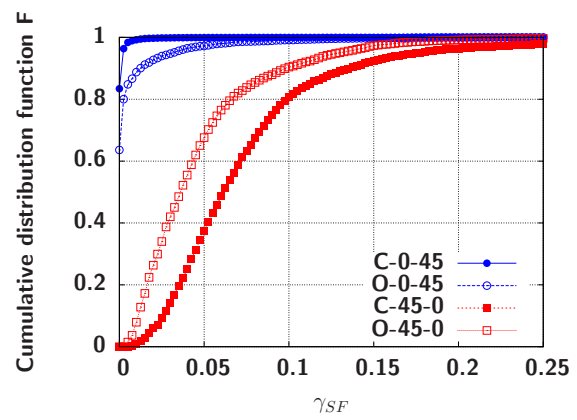

(f) $\Delta E / 2=0.5 \%$

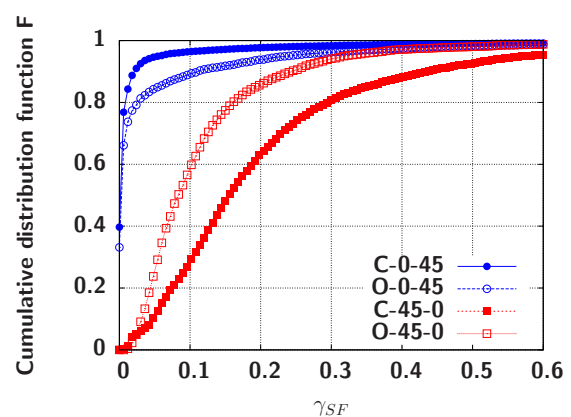

(g) $\Delta E / 2=1.0 \%$

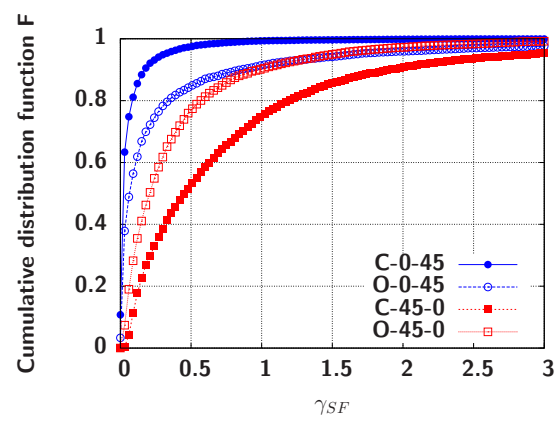

(h) $\Delta E / 2=2.0 \%$

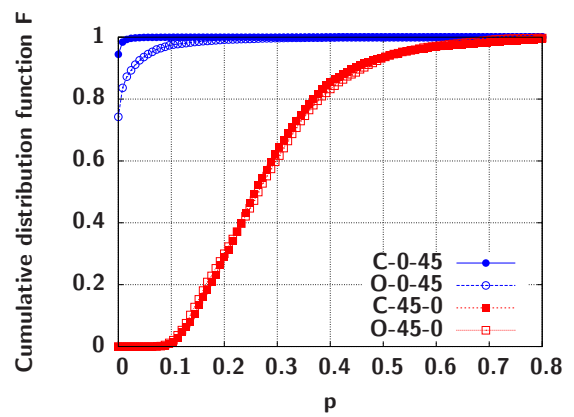

(i) $\Delta E / 2=0.3 \%$

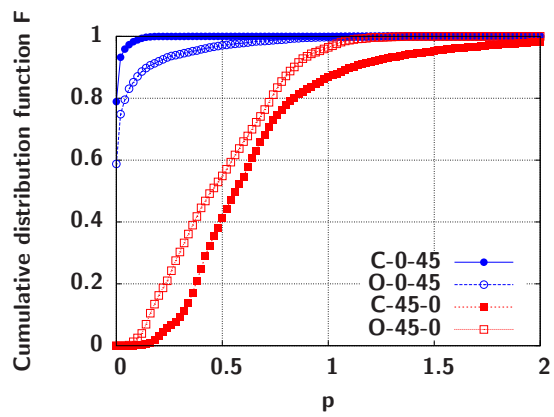

(j) $\Delta E / 2=0.5 \%$

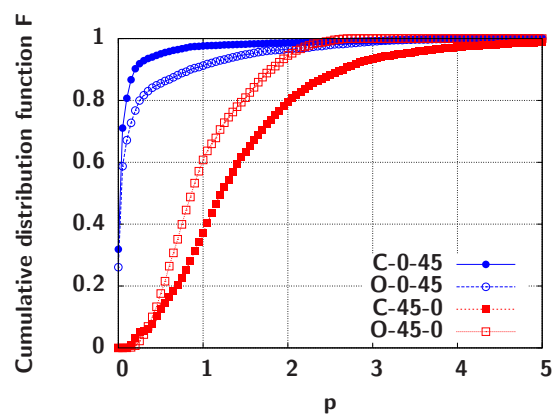

(k) $\Delta E / 2=1.0 \%$

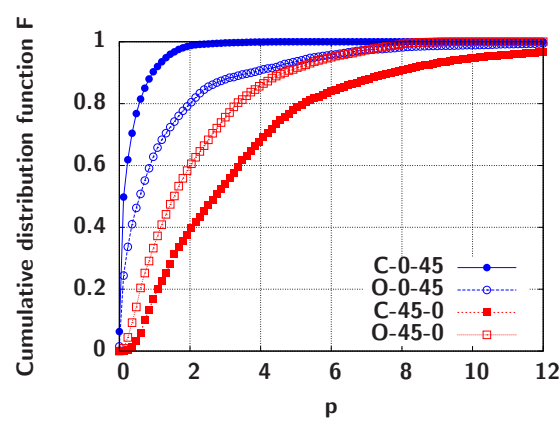

(l) $\Delta E / 2=2.0 \%$

Figure 16: Cumulative distribution functions of fatigue criteria in the central grain of clusters for different loading amplitudes (gauss point values). 
[2] H. Kitagawa, S. Takahashi, Applicability of fracture mechanics to very small cracks or the cracks in the early stage, in: ICM-2, ASM, 1976, pp. 627-631.

[3] M. W. Brown, Interfaces between short, long, and nonpropagating cracks, in: K. J. Miller, E. R. de los Rios (Eds.), The Behaviour of Short Fatigue Cracks, 1986, pp. 423-439.

[4] S. Pearson, Initiation of fatigue cracks in commercial aluminium alloys and the subsequent propagation of very short cracks, Engng Fracture Mechanics 7 (1975) 235-247.

[5] K. J. Miller, The short crack problem, Fatigue and Fracture of Engng Mat and Struct 5 (1982) 223-232.

[6] P. Lukas, Fatigue Crack nucleation and Microstructure, in: ASM Handbook : Fatigue and Fracture, Vol. 19, ASM International, 1996, pp. 96-109.

[7] U. Essmann, U. Gösele, H. Mughrabi, A model of extrusions and intrusions in fatigued metals: I. Point-defect production and the growth of extrusions, Philosophical Magasine 44 (1981) 405-426.

[8] Z. S. Basinski, R. Pascual, S. J. Basinski, Low amplitude fatigue of copper single crystals-I. The role of the surface in fatigue failure, Acta Metall. 31 (1983) 591-602.

[9] M. Mineur, P. Villechaise, J. Mendez, Influence of the crystalline texture on the fatigue behavior of a $316 \mathrm{~L}$ austenitic stainless steel, Material Science and Engineering A286 (2000) 257-268.

[10] C. Blochwitz, J. Brechbühl, W. Tirschler, Analysis of activated slip systems in fatigued nickel polycrystals using the EBSDtechnique in the scanning electron microscope, Material Science and Engineering A210 (1996) 42-47.

[11] C. Blochwitz, R. Richter, Plastic strain amplitude dependent surface path of microstructurally short fatigue cracks if facecentred cubic metals, Material Science and Engineering A267 (1999) 120-129.

[12] C. Blochwitz, R. Richter, W. Tirschler, K. Obrtlik, The effect of local textures on microcrack propagation in fatigued F.C.C. metals, Material Science and Engineering A224-236 (1997) 563566.

[13] K. S. Chan, J. Lankford, The role of microstructural dissimilitude in fatigue and fracture of small cracks, Acta Metall. 36 (1988) 193-206.

[14] T. H. Lin, K. K. F. Wong, N. J. Teng, S. R. Lin, Micromechanic analysis of fatigue band crossing grain boundary, Material Science and Engineering A246 (1998) 169-179.

[15] W. H. Kim, C. Laird, Crack nucleation and stage I propagation in high strain fatigue-I. Microscopic and interferometric observations, Acta Metall. 26 (1978) 789-799.

[16] W. H. Kim, C. Laird, Crack nucleation and stage I propagation in high strain fatigue-II. Mechanism, Acta Metall. 26 (1978) 789-799.

[17] E. Sackett, L. Germain, M. Bache, Crystal plasticity, fatigue crack initiation and fatigue performance of advanced titanium alloys, Int. J. Frac 29 (2007) 2015-2021.

[18] X.-L. Wang, Y. Wang, A. Stoica, D. Horton, H. Tian, P. Liaw, H. Choo, J. Richardson, E. Maxey, Inter- and intragranular stresses in cyclically-deformed 316 stainless steel, Material Science and Engineering 399 (2005) 114-119.

[19] G. P. Potirniche, S. R. Daniewicz, Finite element modeling of microstructurally small cracks using single crystal plasticity, Int. J. Fatigue 25 (2003) 877-884.

[20] V. P. Bennett, D. L. McDowell, Polycrystal orientation distribution effects on microslip in high cycle fatigue, Int. J. Fatigue 25 (2003) 27-39.

[21] R. Lillbacka, E. Johnson, M. Ekh, A model for short crack propagation in polycrystalline materials, Engng Fracture Mechanics 73 (2006) 223-232.

[22] F. P. E. Dunne, D. Rugg, A. Walker, Lengthscale-dependent, elastically anisotropic, physically-based HCP crystal plasticity: Application to cold-dwell fatigue in Ti alloys, Int. J. of Plasticity 23 (2007) 1061-1083.

[23] F. P. E. Dunne, A. J. Wilkinson, R. Allen, Experimental and computational studies for low cycle fatigue crack nucleation in a polycrystal, Int. J. of Plasticity 23 (2007) 273-295.
[24] G. Venkatramani, S. Ghosh, M. Mills, A size-dependent crystal plasticity finite-element model for creep and load shedding in polycrystalline titanium alloys, Acta Mat. 55 (2007) 3971-3986.

[25] I. Simonovski, L. Cizelj, The influence of grain's crystallographic orientations on advancing short crack, Int. J. Fatigue 29 (2007) 2005-2014.

[26] I. Simonovski, K. F. Nilsson, L. Cizelj, The influence of crystallographic orientation on crack tip displacements of microstructurally small, kinked crack crossing grain boundary, Computational Materials Science 39 (2007) 817-828.

[27] I. Simonovski, K. F. Nilsson, L. Cizelj, Crack tip displacement of microstructurally small cracks in $316 \mathrm{~L}$ steel and their dependence on crystallographic orientations of grains, Fatigue and Fracture of Engng Mat and Struct 30 (2007) 463-478.

[28] I. Simonovski, L. Cizelj, Grain scale model of small cracks, in: 15th International Conference on Nuclear Engineering, Nagoya, Japan, 2007.

[29] K.-S. Cheong, M. Smillie, D. Knowles, Predicting fatigue crack initiation through image-based micromechanical modeling, Acta Mat. 55 (2007) 1757-1768.

[30] C. P. Przybyla, D. L. McDowell, Microstructure-sensitive extreme value probabilities for high cycle fatigue of Ni-base superalloy IN100, Int. J. of Plasticity 26 (2010) 372-394.

[31] L. Méric, G. Cailletaud, Single crystal modeling for structural calculations. Part 2: Finite element implementation, J. of Engng. Mat. Technol. 113 (1991) 171-182.

[32] M. Sauzay, Effet de l'anisotropie élastique cristalline sur la distribution des facteurs de Schmid à la surface des polycristaux, C.R. Mécanique 334 (2006) 353-361.

[33] J. Besson, G. Cailletaud, J.-L. Chaboche, S. Forest, Nonlinear Mechanics of Materials, Springer, 2009.

[34] N. Zouhal, A. Molinari, L. S. Tóth, Elastic-plastic effects during cyclic loading as predicted by the Taylor-Lin model of polycrystal elasto-viscoplasticity, Int. J. of Plasticity 12 (3) (1996) 343-360.

[35] A. S. Argon, Effect of surfaces on fatigue crack initiation, in: O. F. Devereux, A. J. McEvily (Eds.), Proc. Corrosion Fatigue, NACE, 1972, p. 176.

[36] A. Fatemi, D. F. Socie, A critical plane approach to multiaxial fatigue damage including out-of-phase loading, Fatigue and Fracture of Engng Mat and Struct 11 (1988) 145-165.

[37] K. Dang Van, Sur la résistance à la fatigue des métaux, Sciences et techniques de l'armement 47 (1973) 647-722.

[38] F. Barbe, S. Forest, G. Cailletaud, Intergranular and intragranular behavior of polycrystalline aggregates. Part II: Results, Int. J. of Plasticity 17 (4) (2001) 537-566. 